\title{
Modeling transcranial magnetic stimulation from the induced electric fields to the membrane potentials along tractography- based white matter fiber tracts
}

\author{
Nele De Geeter, Luc Dupré and Guillaume Crevecoeur \\ Department of Electrical Energy, Systems and Automation, Ghent University, Technologiepark 913, \\ B-9052 Zwijnaarde, Belgium \\ E-mail: Nele.DeGeeter@UGent.be
}

Received 9 September 2015, revised 3 February 2016

Accepted for publication 10 February 2016

Published 2 March 2016

\begin{abstract}
Objective. Transcranial magnetic stimulation (TMS) is a promising non-invasive tool for modulating the brain activity. Despite the widespread therapeutic and diagnostic use of TMS in neurology and psychiatry, its observed response remains hard to predict, limiting its further development and applications. Although the stimulation intensity is always maximum at the cortical surface near the coil, experiments reveal that TMS can affect deeper brain regions as well. Approach. The explanation of this spread might be found in the white matter fiber tracts, connecting cortical and subcortical structures. When applying an electric field on neurons, their membrane potential is altered. If this change is significant, more likely near the TMS coil, action potentials might be initiated and propagated along the fiber tracts towards deeper regions. In order to understand and apply TMS more effectively, it is important to capture and account for this interaction as accurately as possible. Therefore, we compute, next to the induced electric fields in the brain, the spatial distribution of the membrane potentials along the fiber tracts and its temporal dynamics. Main results. This paper introduces a computational TMS model in which electromagnetism and neurophysiology are combined. Realistic geometry and tissue anisotropy are included using magnetic resonance imaging and targeted white matter fiber tracts are traced using tractography based on diffusion tensor imaging. The position and orientation of the coil can directly be retrieved from the neuronavigation system. Incorporating these features warrants both patient- and case-specific results. Significance. The presented model gives insight in the activity propagation through the brain and can therefore explain the observed clinical responses to TMS and their inter- and/or intra-subject variability. We aspire to advance towards an accurate, flexible and personalized TMS model that helps to understand stimulation in the connected brain and to target more focused and deeper brain regions.
\end{abstract}

S Online supplementary data available from stacks.iop.org/jne/13/026028/mmedia

Keywords: computational modeling, diffusion tensor imaging (DTI), electric field, membrane potential, tractography, transcranial magnetic stimulation (TMS)

(Some figures may appear in colour only in the online journal)

\section{Introduction}

Transcranial magnetic stimulation, abbreviated as TMS, is a non-invasive technique that can modulate the brain activity. A coil generating a time-varying magnetic field is held above the head. This induces an electric field in the brain that can excite or suppress temporarily the activity of certain brain regions by altering the membrane potentials of the neurons. In 
recent years, this safe and well tolerated brain stimulation technique has established itself as a promising diagnostic tool and alternative treatment for a broad range of neurological and psychiatric disorders (Ilmoniemi et al 1999, Fox et al 2014). Since TMS has the ability to modify the brain state, it is also a powerful tool to explore specific brain functions. It can temporarily disrupt the normal behavior of a given cortical area, thus creating a 'virtual brain lesion' (Pascual-Leone et al 2000). This enables, for example, the mapping of critical motor and speech-related areas of the cortex prior to neurosurgery (Picht et al 2013, Krieg et al 2014). Although the efficacy of TMS has already been demonstrated, its effect remains poorly understood and is therefore hard to predict (Sack and Linden 2003, Hoogendam et al 2010). Many clinical and psychological studies that employ TMS report a high variability of the response on brain functions (Maeda et al 2000, Loo and Mitchell 2005, Lioumis et al 2009, Casarotto et al 2010), where limited parameter changes in the TMS procedure lead to drastic changes in the clinical or psychological outcome.

Up to now, much research on modeling TMS has already been performed. The induced electric field is calculated (Thielscher and Kammer 2004, Salinas et al 2007, De Geeter et al 2012, Janssen et al 2013, Krieg et al 2015) and, related to this field, the activation function and stimulation mechanisms are studied (Lu et al 2008, Opitz et al 2011, Pashut et al 2011, Salvador et al 2011, De Geeter et al 2014, Shahid et al 2014). However, we believe that these electromagnetic computations are not sufficient to fully understand the observed clinical response to TMS, to explain its reported variability and thus to optimize the currently existing devices. Gaining knowledge of the physiological changes occurring within the brain during stimulation is, to our opinion, essential as well and therefore previous field modeling should be coupled with models describing neurophysiology. The applied electric field interacts with the voltage-gated ion channels at the plasma membrane of neurons, in accordance with the HodgkinHuxley dynamics (Hodgkin and Huxley 1952), and alters the membrane potentials. Once they depolarize sufficiently, the neurons will communicate through the conduction of neuronal signals, i.e., action potentials. While applying TMS, a change in brain activity is not only reported in cerebral areas nearby the coil, but also in distant regions (Kähkönen et al 2005, Hannula et al 2010, Ferreri et al 2011). Moreover, TMS can cause responses lasting longer than the duration of the stimulation itself (Thut and PascualLeone 2010). This spread of TMS-evoked activity has been demonstrated with electroencephalography (Ilmoniemi et al 1997) and functional magnetic resonance imaging (fMRI) data (Bestmann et al 2004, Fox et al 2012). It indicates that computational TMS models need to account for spatial and temporal effects of TMS in the brain. This paper therefore elaborates on the coupling of the induced electric field and the neuronal response to TMS, so to compute the spatio-temporal behavior of the membrane potentials.
As shown by Heller and van Hulsteyn (1992), the generated electromagnetic fields are always maximal at the cortical surface, close to the TMS coil, and penetrate into subcortical structures up to a limited depth. The activation depth of a figure-of-eight coil is generally limited to $2.0 \mathrm{~cm}$ beneath the scalp (Zangen et al 2005). Researchers have been investigating the use of conductive shields, placed between the coil and the subject's head (Davey and Riehl 2006, Kim et al 2006). These shields can inhibit or divert the generated magnetic field, leading to more focused stimulation and improved field penetration. Complex coil designs (Zangen et al 2005, Crowther et al 2011, Deng et al 2013) have also been developed where the rate of decay from the surface is attenuated, such that the percentage of electric field intensity is increased in deeper brain regions, relative to the maximum field at the cortex. However, experiments reveal that standard TMS coils such as the figure-of-eight coil possess the ability to affect these deeper brain regions as well (Bestmann et al 2004, Hannula et al 2010, Ferreri et al 2011). This might be attributed to the network of white matter fibers present in the brain, connecting cortical and subcortical structures. TMS can depolarize the membrane potentials and initiate action potentials within the superficial neural tissue underneath the coil, which in turn can propagate along neural pathways towards deeper regions.

This paper uses the independent impedance method (De Geeter et al 2011a, 2011b, 2012) for the electric field calculations, which serve as the input to compute the spatiotemporal behavior of the membrane potentials on neural pathways. The latter computations are based on the compartmental cable equation (Salvador 2009). We work towards realistic and clinical relevant simulations. Personalized head models are used and include realistic geometry and frequency-dependent anisotropic tissue properties, i.e., conductivity and permittivity, based on T1-weighted and diffusion-weighted MRI, the latter also known as diffusion tensor images (DTI). DTI exploits the directional dependent diffusion of water molecules in the brain, with the principal diffusion direction corresponding to the predominant orientation of white matter fibers (Jones and Leemans 2011). Including these features is important, since anisotropy and tissue heterogeneity can significantly alter the electric field distribution (Miranda et al 2003, De Lucia et al 2007, Miranda et al 2007). The coil configuration can be chosen freely and is specified by the coil shape, stimulation pulse and position to the head. Nowadays, many hospitals and research centers possess neuronavigated TMS devices, by which the coil is positioned based on individual MRI with increased accuracy and reproducibility compared to manual coil positioning (Julkunen et al 2009, Lioumis et al 2009). Our developed solver can directly retrieve these monitored coil positions and orientations relative to the subject's head from the neuronavigation system. DTI-based fiber tractography is applied to reconstruct the realistic 3D neural trajectories (Basser et al 2000, Leemans et al 2009). Close to the brain region of interest (ROI) that is targeted with the TMS coil, bundles of grouped white matter fibers, called tracts, are selected. The induced electric field is mapped 
along the trajectories of these tracts and serves as the input for the considered stimulation mechanisms, as Shahid et al (2014) did for transcranial direct current stimulation. We describe the response of these mechanisms on the membrane potential by a compartmental cable equation. All tracts are assumed to be myelinated neurons, containing sections representing dendrites, soma, axon hillock, initial segment and alternating Ranvier nodes and myelinated internodes, and are modeled with passive and active membrane properties.

The developed flexible and personalized solver models TMS from the macroscopic electric fields in the brain to the membrane potentials on microscopic neuronal level. The novelty of this work lies in the use of DTI data to reconstruct white matter fiber trajectories, which is a more realistic approach than in most studies published so far (Goodwin and Butson 2015) and a valuable step towards fully case- and patient-specific results. We provided a model for electrical activity propagation through the brain. It has the main advantage that it apprehends how TMS can reach deeper regions, which may lead to better targeting and hence more effective brain stimulation. Moreover, it might explain why the effect of TMS is not fixed, but determined by the brain state such as the membrane resting potentials and channel properties during stimulation (Silvanto and Muggleton 2008, Pasley et al 2009) and the variability of the neural network both among healthy (Cahn et al 2003) and patient populations (Brighina et al 2002).

Our approach is encouraged by the findings of Fox et al (2012, 2013, 2014). The authors consider the human brain as a complex interconnected grid as well and state that TMS can affect an area of the brain network that is not necessarily at the targeted site. When studying the most effective TMS target brain regions of 14 neurological and psychiatric diseases, Fox et al (2014) found that these different sites are often nodes within the same network. Using a database of fMRI, correlations in spontaneous brain activity, and consequently brain networks of functional connectivity were studied. Fox and colleagues investigated the brain network spreading out from the sites stimulated by deep brain stimulation for a certain disorder, and showed all of the connections reaching up to the surface of the brain are matching the corresponding TMS targets. Moreover, Sollmann et al (2015) reported very recently a clinical case where DTIbased fiber tracking and navigated TMS are combined to visualize language pathways prior to the surgery of a brain tumor patient. Sollmann and colleagues could identify language-related white matter fiber tracts that were confirmed with intraoperative mapping by direct cortical stimulation. These studies (Fox et al 2012, 2013, 2014, Sollmann et al 2015) confirm the importance of identifying the brain network. However, they mention clinical observations, whereas we aim to describe and support them with high fidelity computational modeling.

This paper considers the spatio-temporal variation of the membrane potentials due to stimulation of the primary motor cortex (M1) with a standard figure-of-eight coil. We first focus on a single tract to elaborate on the coupled model, i.e., how the induced electric field changes the membrane potential of the reconstructed neural trajectory and possibly leads to the generation and propagation of an action potential. The influence of an increasing stimulator output is investigated, together with a sensitivity analysis of the not well-known neural parameter values on the stimulation threshold. Finally, we calculate the spread of activation along a group of 54 selected tracts near the targeted site.

\section{Methodology}

\subsection{Personalized head model with traced fiber tracts}

A T1-weighted image was acquired from a healthy 25 yearold female volunteer (Philips Achieva 3.0T). SPM8 (Friston et al 2007) was used to segment this image into tissue probability maps. Based on these maps, a head model was constructed $\left(\right.$ FOV $192 \times 236 \times 206 \mathrm{~mm}^{3}$ and matrix size $96 \times 126 \times 110$ ), surrounded with air and segmented into scalp, skull and the cerebral tissues cerebrospinal fluid (CSF), gray and white matter as visualized in figure 1 . The electrical properties, i.e., conductivity and permittivity, of these tissues vary naturally with frequency and their isotropic values were obtained from the four-Cole-Cole model (Cole and Cole 1941, Gabriel et al 1996). The electrical properties that are assigned to the tissues for the applied fundamental stimulation frequency of $4.348 \mathrm{kHz}$ (pulse width $=230 \mu \mathrm{s}$ ) are listed in table 1 . Note, the skull is modeled as homogeneous without distinguishing between compact and spongy bone (Dannhauer et al 2011).

In addition, a DTI data set of the same subject was acquired with 60 diffusion directions and co-registered to the T1-weighted image. Anisotropic electrical properties were computed from the diffusion tensors as explained in (De Geeter et al 2012). To reconstruct the fiber trajectories in the subject's brain, we used the graphical toolbox ExploreDTI (Leemans et al 2009). Whole brain tractography (Basser et al 2000) was performed with a step size of $1 \mathrm{~mm}$, a fractional anisotropy threshold of 0.2 , a bending angle threshold of $30^{\circ}$ and a minimum fiber length of $50 \mathrm{~mm}$. From this extensive tractogram, we will extract a group of relevant white matter fiber tracts by applying the ROI method. To determine this ROI, we first need to calculate the electric field distribution induced by the TMS coil.

\subsection{Electromagnetism: macroscopic (effective) electric field distribution}

We simulate the effect of a single biphasic pulse stimulation (pulse width $230 \mu \mathrm{s}$ ) delivered to the hand area of the left primary motor cortex, with a $70 \mathrm{~mm}$ figure-of-eight coil (Nexstim Ltd, Helsinki, Finland) positioned according to figure 1. The stimulator output (peak excitation current's

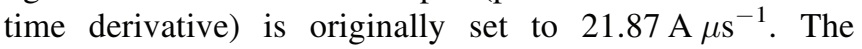
excitation current flowing through the TMS coil causes a magnetic field, described by Biot-Savart's law. The time variation of this magnetic field induces an electric field in the 

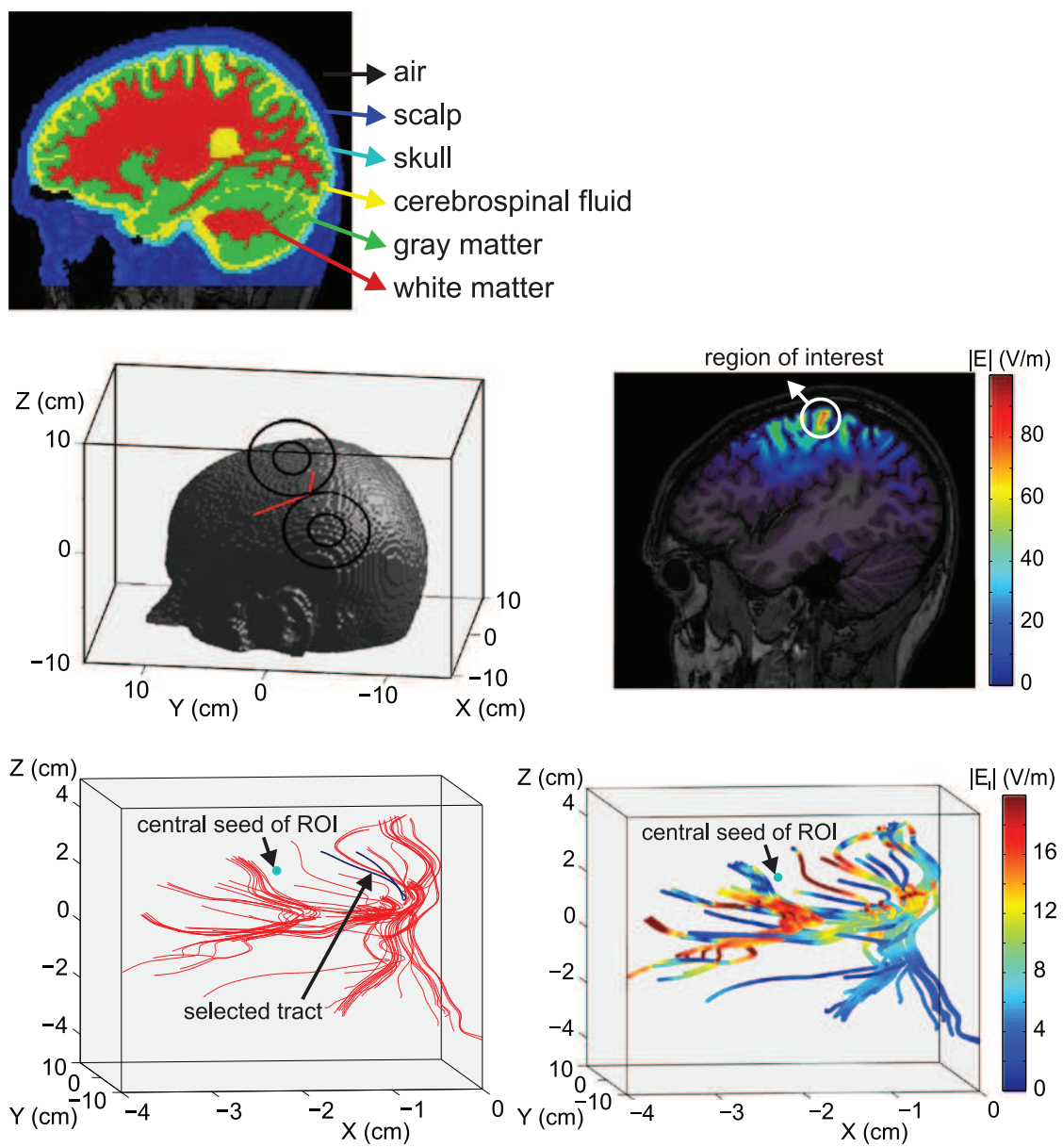

Figure 1. Segmented head model based on T1-weighted MRI. Coil location and orientation for stimulation of the left M1 hand area. The stimulator output is set to $21.87 \mathrm{~A} \mu \mathrm{s}^{-1}$. The resulting induced electric field amplitude distribution on which the region of interest is defined. Near the central seed of this ROI, 54 fiber tracts are considered of which one, indicated in blue, is selected for the study in depth. The effective electric field amplitude along the neural tracts. These figures are adapted from (De Geeter et al 2014).

Table 1. Electrical properties according to the four-Cole-Cole model (Cole and Cole 1941, Gabriel et al 1996). The permittivity is expressed relative to the permittivity of vacuum $\epsilon_{0}=(1 / 36 \pi)$ $10^{-9} \mathrm{~F} \mathrm{~m}^{-1}$.

\begin{tabular}{lcc}
\hline Tissue & Permittivity $\left(\mathrm{F} \mathrm{m}^{-1}\right)$ & Conductivity $\left(\mathrm{S} \mathrm{m}^{-1}\right)$ \\
\hline Air & $1.0005 \epsilon_{0}$ & 0.0 \\
Scalp (skin) & $30168 \epsilon_{0}$ & 0.0012 \\
Skull (bone) & $933.62 \epsilon_{0}$ & 0.0203 \\
CSF & $109 \epsilon_{0}$ & 2.0 \\
Gray matter & $48070 \epsilon_{0}$ & 0.1083 \\
White matter & $23152 \epsilon_{0}$ & 0.0662 \\
\hline
\end{tabular}

material below the coil. For the considered case study, the induced electric field in the whole brain is computed using the anisotropic independent impedance method (De Geeter et al 2014).

Since the cerebral tissues are of main interest, only the induced electric field distribution within those voxels is imaged in figure 1. It is plotted at the time instant that the stimulation pulse begins, corresponding with maximum induced electric fields, as can be seen from the typical biphasic waveforms of figure 2. The magnetic flux density produced by the coil exhibits the same waveform as the damped sinusoidal excitation current, whereas the induced current density and the induced electric field exhibit the same waveform as the excitation current's time derivative. The induced voltage in the brain follows a similar triphasic curve. Neural membranes are most likely to depolarize or hyperpolarize when this voltage is positive or negative, respectively. For the biphasic TMS pulse with a pulse width of $200 \mu \mathrm{s}$ of figure 2, depolarization will occur first $( \pm 0-50 \mu \mathrm{s})$, followed by a larger hyperpolarization $( \pm 50-150 \mu \mathrm{s})$. Since activation may only occur when the membrane is sufficiently depolarized, the threshold for neuronal activation would thus be lower if the current's polarity would be reversed.

Based on the electric field distribution, the central seed ROI, depicted as the blue dot in figure 1 , is defined using the position of the weighted mean. Within its $15 \mathrm{~mm}$ range, the ROI box is created. Those tracts that traverse this box are identified from the whole-brain tractogram, and reduced to a total of 54 neural fiber tracts, shown in figure 1. For more details on the applied selection criteria, we refer to (De Geeter et al 2014). 


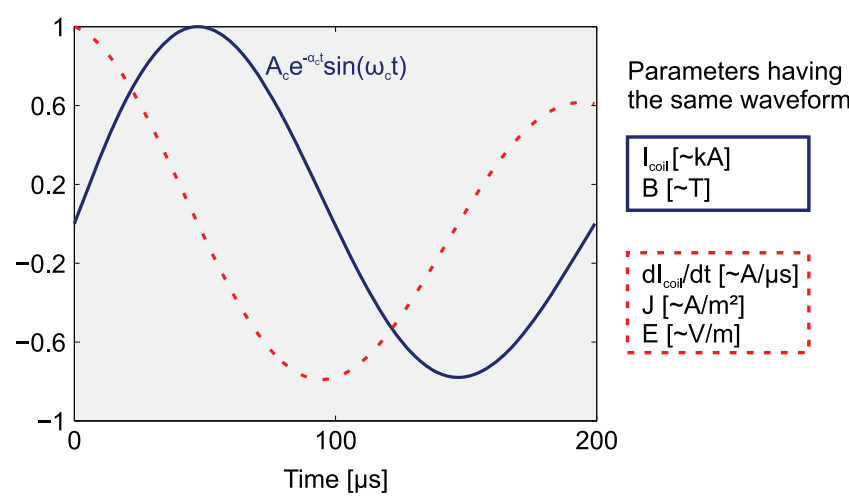

Figure 2. Typical normalized waveforms during a biphasic TMS pulse of the damped sinusoidal excitation current $I_{\text {coil }}$, the magnetic flux density $B$, the excitation current's time derivative $\mathrm{d} I_{\text {coil }} / \mathrm{d} t$, the induced current density $J$ and the induced electric field $E$ and their corresponding order of magnitudes.

The component of the induced electric field $E$ tangent to the neural trajectory $\mathbf{I}=l \mathbf{1}_{l}$ effectively contributes to neuronal stimulation and is therefore called the effective electric field $E_{l}=\mathbf{E} \cdot \mathbf{1}_{l}$. Initially, the directional derivative of this effective electric field along the fiber tract $-\lambda^{2} \partial E_{l} / \partial l$ was assumed to describe the effect of stimulation by electromagnetic induction on the membrane potential (Rattay 1986), with $\lambda$ being the space constant. Silva et al (2008) later introduced two additional stimulation mechanisms; $-\lambda E_{l}$ occurring at terminations and sharp bends of the fiber, and $-\lambda \Delta E / 2$ at the interface between tissues that have different electrical conductivities, such as gray and white matter $\left(0.1083 \mathrm{~S} \mathrm{~m}^{-1}\right.$ and $0.0662 \mathrm{~S} \mathrm{~m}^{-1}$, respectively, see table 1). The effective electric field is computed along the 54 realistic neural fiber trajectories, as shown in figure 1.

\subsection{Neurophysiology: spatio-temporal behavior of membrane potentials}

To model the excitability of the fiber tracts and the possible generation and propagation of action potentials, we consider the membrane potential that expresses the ion balance in the neurons. We distinguish a passive and an active response to stimulation. In reality, the myelinated neural fibers contain passive dendrites, a passive soma, an active axon hillock, an active initial segment and alternating passive myelinated internodes and active Ranvier nodes. We model them as compartmental neurons with passive and active membrane properties, as schematically illustrated in figure 3 .

They are divided into several cylindrical compartments or segments with diameter $d$ and a total length $L$, which are divided in smaller space steps of length $\Delta l$. The behavior of their membrane potential $V$ can be computed as a function of time $t$ and space $l$ with the following compartmental cable

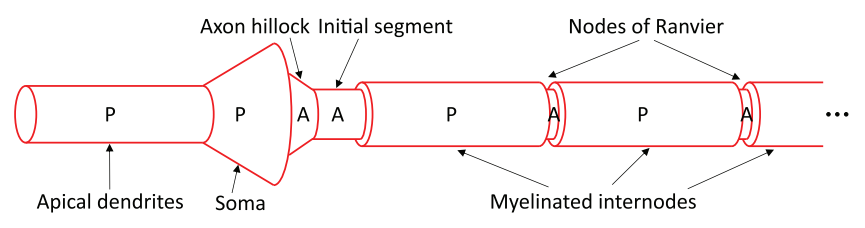

Figure 3. Schematic representation of the modeled myelinated neuron with the different passive $(\mathrm{P})$ and active (A) compartments.

equation (Nagarajan et al 1993, Salvador 2009)

$$
\begin{aligned}
C_{\mathrm{m}}^{*} \frac{\partial V(l)}{\partial t}+I_{\mathrm{ion}}(l)= & G_{\mathrm{a}}^{*}(V(l-\Delta l)-2 V(l)+V(l+\Delta l)) \\
& +G_{\mathrm{a}}^{*} \Delta l E_{l}(l-\Delta l)-G_{\mathrm{a}}^{*} \Delta l E_{l}(l) .
\end{aligned}
$$

The membrane's capacitance $C_{\mathrm{m}}^{*}$ and axial conductance $G_{\mathrm{a}}^{*}$ are

$$
\begin{aligned}
C_{\mathrm{m}}^{*} & =\pi d \Delta l C_{\mathrm{m}}, \\
G_{\mathrm{a}}^{*} & =\frac{\pi d^{2}}{4 \Delta l R_{\mathrm{a}}}
\end{aligned}
$$

with $C_{\mathrm{m}}$ the capacitance per unit area and $R_{\mathrm{a}}$ the resistivity of the axoplasm. The superscript $*$ refers to the fact that the values are not per unit area. For the passive components, the ionic current equals

$$
I_{\text {ion }}=\pi d \Delta l G_{\mathrm{m}}\left(V(l)-V_{\mathrm{r}}\right)
$$

whereas it describes a more complex behavior for the active components (Wesselink et al 1999)

$$
\begin{aligned}
I_{\text {ion }}= & \pi d \Delta l\left(G_{\mathrm{Na}} m^{3} h\left(V(l)-V_{\mathrm{Na}}\right)\right. \\
& \left.+G_{\mathrm{K}} n^{4}\left(V(l)-V_{\mathrm{K}}\right)+G_{\mathrm{L}}\left(V(l)-V_{\mathrm{L}}\right)\right)
\end{aligned}
$$

with $V_{\mathrm{r}}$ the membrane's resting potential and $G_{\mathrm{m}}$ the membrane conductance per unit area. $V_{i}(i=\mathrm{Na}, \mathrm{K}, \mathrm{L})$ are the Nernst potentials for the sodium, potassium and leakage channels and $G_{i}$ the conductances per unit area. The sodium current is controlled by two types of fictional gates; one for activation $m$ and one for inactivation $h$. The activation gate $m$ contributes to the fast opening of the sodium channel with increasing voltage, whereas $h$ contributes to the relatively slow closing. The factor $m^{3} h$ represents the probability that the sodium channel is open with a conductance per unit area $G_{\mathrm{Na}}$. The potassium current is controlled by a single type of fictional gates; one for activation $n$. The gate has to be in an open configuration to allow an outward current of potassium ions. The temporal changes of these gates are described by

$$
\frac{\mathrm{d} j}{\mathrm{~d} t}=\alpha_{j}(1-j)-\beta_{j} j
$$

with $j=m, h, n$ and $\alpha_{j}$ and $\beta_{j}$ the voltage-dependent transition rates. For the practical implementation of the 
Table 2. Space step, space constant and time constant of the different passive (P) and active (A) neural segments, based on human sensory fiber data (Wesselink et al 1999, Salvador 2009).

\begin{tabular}{lccc}
\hline Segment & $\Delta l(\mu \mathrm{m})$ & $\lambda(\mu \mathrm{m})$ & $\tau(\mu \mathrm{s})$ \\
\hline Dendrite (P) & 100 & 1490 & 10260 \\
Soma (P) & 8 & {$[1490-4080]$} & 10260 \\
Axon hillock (A) & 1 & {$[123-87]$} & 46.54 \\
Initial segment (A) & 2 & 87 & 46.54 \\
Myelin. internode (P) & $\pm 100^{\mathrm{a}}$ & 8700 & 500 \\
Ranvier node (A) & 0.15 & 87 & 46.54 \\
\hline
\end{tabular}

${ }^{a}$ Depending on the length of the tract.

compartmental cable equation and applied parameter values, we refer to appendix.

There is a difference of several orders of magnitude between the lengths of the various compartments. For example, the length of one Ranvier node is approximately 1000 times smaller than that of one myelinated internode, see table A.1. To avoid excessive use of computational memory, we implement a variable space step such that all segments are divided in 10 steps. A node of Ranvier is discretized with a very fine space step $\Delta l$ of $0.15 \mu \mathrm{m}$, while myelinated internodes have a spatial grid of about $100 \mu \mathrm{m}$, depending on the exact length of the neural fiber tract. This way, the number of tract points is reduced from 500000 (when a fixed $\Delta l$ of $0.15 \mu \mathrm{m}$ is used) to about 1500 for a fiber tract with an average length of $75 \mathrm{~mm}$. The time step $\Delta t$ is $1 \mu \mathrm{s}$. Table 2 lists the applied space steps of the neural segments and their space and time constants, calculated as:

$$
\begin{gathered}
\lambda=\sqrt{\frac{d}{4 R_{\mathrm{a}} G_{\mathrm{m}}}}, \\
\tau=\frac{C_{\mathrm{m}}}{G_{\mathrm{m}}} .
\end{gathered}
$$

Individual neurons, and even different patches of the membrane within a given neuron, can have different space constants. When the membrane conductance is high, ions (that cause the potential change) can leak through the membrane via open channels, resulting in a shorter space constant. When a neuron has a large diameter, the ions can flow easily along the axon and the axial resistance will be low, leading to a larger space constant. This explains the different $\lambda$ values between active and passive segments and its range for those segments with variable diameter. The conductance of the active components, equal to $G_{\mathrm{Na}} m^{3} h+G_{\mathrm{K}} n^{4}+G_{\mathrm{L}}$, is computed based on the initial values of the gates (A.13)(A.15). As these gating variables vary in time depending on the voltage, the conductance and consequently the space constant change as well. According to Rattay (1999), the compartmental space step should be smaller than $\lambda / 4$ to obtain an error of the order of $1 \%$ compared to the solution of

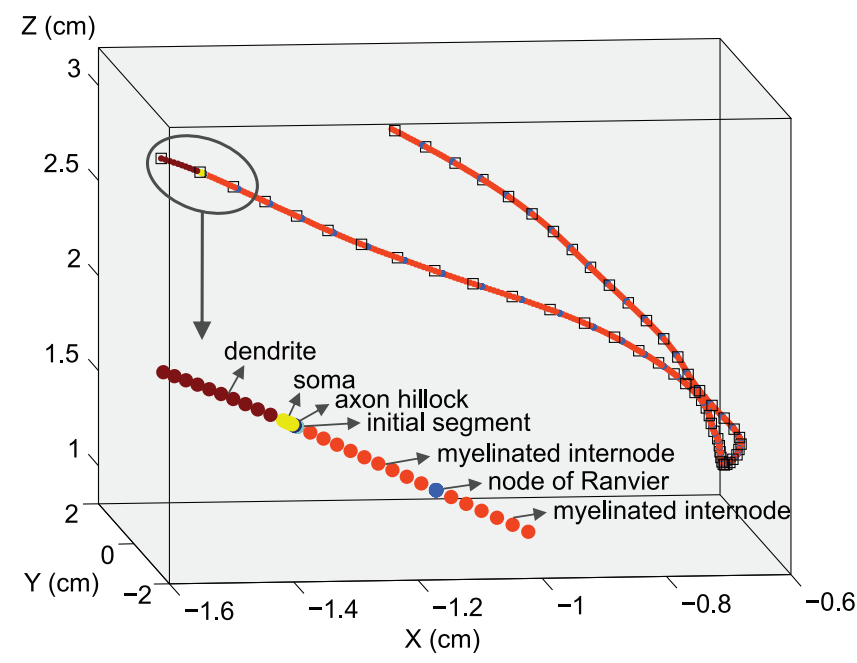

Figure 4. Orientation of the selected tract with its corresponding segments. The black squares indicate the original tract coordinates with $1 \mathrm{~mm}$ step. Trilinear interpolation is used to convert the fiber tract from this coarse spatial grid to the more fine and variable mesh. A close-up from the first 65 tract points with variable fine step and their corresponding type of segment is further shown.

the continuous cable equation. The applied $\Delta l$ and $\lambda$ values of all neural segments easily meet this requirement, see table 2 .

\section{Results and discussion}

We simulate (a) TMS response(s) for targeting the left M1 hand area using a realistic head model, as detailed in section 2.1. Contrary to previous research (De Geeter et al 2014), the effect of stimulation on the membrane potential is investigated instead of the stimulation mechanisms $-\lambda^{2} \partial E_{l} / \partial l,-\lambda E_{l}$ and $-\lambda \Delta E / 2$. The membrane potential $V(l, t)$ is calculated with equation (1) of section 2.3 on the basis of the induced effective electric field $E_{l}(l, t)$, computed in section 2.2. This coupling of both phenomena enables us to investigate how TMS spreads electrical brain activity along the neural pathways, reconstructed using tractography. Whereas the effective electric field and stimulation mechanisms only occur during the excitation pulse, their effect on the membrane potentials can last longer. Thus with our new approach, we can model the propagation of TMS responses in space and over time.

We first focus on a single white matter fiber tract, more specifically the blue tract of figure 1, which is an association fiber, plotted individually in figure 4 . We demonstrate for this selected tract how, where and when action potentials are generated with increasing stimulator output. We study how sensitive the obtained stimulation threshold is towards the uncertainties of the neural parameter values. Finally, we simulate the spatio-temporal variation of the membrane potentials for all 54 neural tracts near the targeted area. 

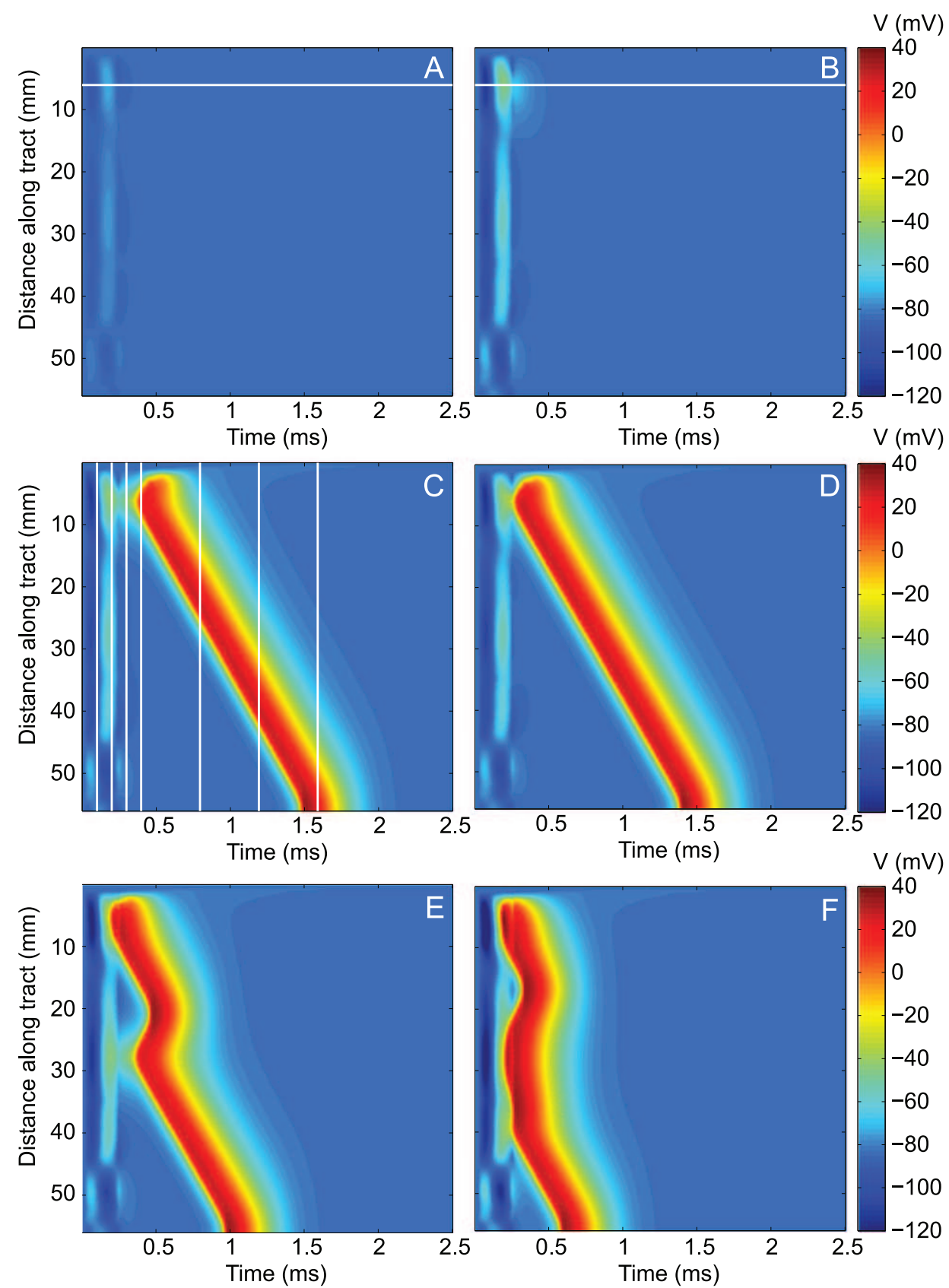

Figure 5. Variation of the membrane potential $V$ in time and distance along the neural tract for increasing stimulation intensity. (A)

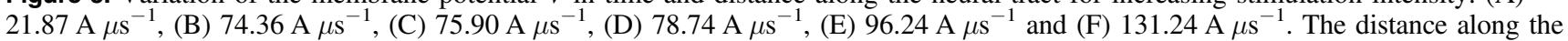
neural tract is measured starting from the point closest to the M1 ROI. To facilitate interpretation, the horizontal white lines of (A) and (B) are depicted in figure 6 as a function of time and the vertical white lines of (C) are plotted in figure 7 as a function of space.

\subsection{Increasing the stimulator output}

The membrane potential is assumed to be initially at rest, at its resting value $V_{\mathrm{r}}$ equal to $-84 \mathrm{mV}$. After $20 \mu \mathrm{s}$, a single biphasic pulse with a pulse width of $230 \mu \mathrm{s}$ is applied. First, the peak excitation current's time derivative is set to

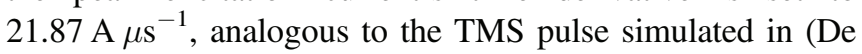
Geeter et al 2014). We gradually increase this stimulator output and the resulting spatio-temporal distributions of the membrane potential are shown in figure 5 .

Case A confirms that a stimulator output of $21.87{\mathrm{~A} \mu \mathrm{s}^{-1}}^{-1}$ is not high enough for the initiation of action potentials within the investigated tract. Only moderate changes of the membrane potential are observed for the duration of the stimulus. Figure 6(A) focuses on the tract point at $l=6.1 \mathrm{~mm}$, indicated with a white horizontal line in figure 5(A). It shows that the induced effective electric field yields a maximum value of $14.3 \mathrm{~V} \mathrm{~m}^{-1}$. First a hyperpolarization from -84 to $-92.3 \mathrm{mV}$ occurs, than a depolarization to $-73.1 \mathrm{mV}$, followed by a hyperpolarization to $-87.9 \mathrm{mV}$. This sequence is the result of the alternating sign of the effective electric field. It corresponds with the fact that $-\lambda E_{1}$ is the dominant stimulation mechanism, as reported in (De Geeter et al 2014). After the 

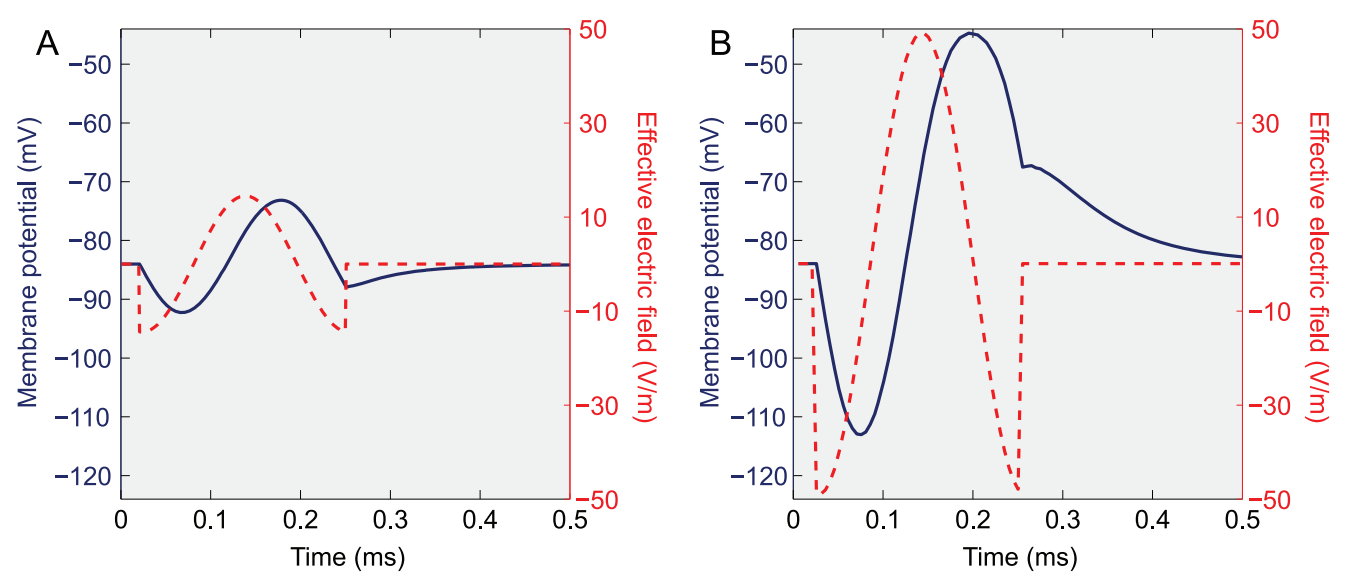

Figure 6. The effective electric fields and corresponding membrane potentials at point $l=6.1 \mathrm{~mm}$ of the selected tract, where the highest potentials are observed. The same information as the white horizontal lines of figures 5(A) and (B) is plotted as a function of time. These results are in agreement with the theoretical waveforms of figure 2 , since $-\lambda E_{1}$ is the dominant stimulation mechanism.

TMS pulse, at $250 \mu \mathrm{s}$, the membrane potential returns to its resting state.

When increasing the stimulator output to $74.36 \mathrm{~A} \mu \mathrm{s}^{-1}$ (case B), a similar wave pattern is produced, see figures $5(\mathrm{~B})$ and 6(B). The effective electric field rises to $48.4 \mathrm{~V} \mathrm{~m}^{-1}$, resulting in a maximum membrane potential of $-44.73 \mathrm{mV}$. After the TMS pulse, a higher and longer transient response is observed than for case A. No action potential was generated up to this point of stimulation. Figure 8(B) displays the corresponding $m, h$ and $n$ values of the fictional gates as a function of time. The open probability of the activation gate for the sodium channel $m$ starts increasing from 0.1 to $0.24 \mathrm{~ms}$, whereas the sodium inactivation variable $h$ decreases and the potassium activation variable $n$ increases to $0.385 \mathrm{~ms}$. It takes approximately $2.5 \mathrm{~ms}$ before they are all returned to their resting values.

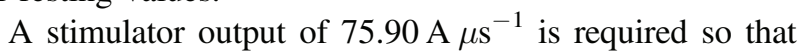
the depolarized membrane exceeds the threshold (case $\mathrm{C}$ ). This stimulation threshold is comparable with the obtained values of Salvador et al (2011). Figure 5(C) shows that an action potential originates at position $l=6.1 \mathrm{~mm}$ (at a node of Ranvier) and time $t=0.38 \mathrm{~ms}$ and progresses along the fiber tract till it reaches the end of the axon at $l=56.1 \mathrm{~mm}$ and $t=1.48 \mathrm{~ms}$. The velocity of the signal can thus be approximated as $45 \mathrm{~m} \mathrm{~s}^{-1}$. Note, this action potential is initiated after the stimulation pulse, that ends at $t=0.25 \mathrm{~ms}$. The variation of the membrane potential and thus the propagation of the action potential is presented in figure 7 as a function of the distance along the tract, at different time points, which are indicated as white vertical lines in figure 5(C). During the stimulus, the membrane potential varies according to the expected pattern, i.e., mainly hyperpolarization for negative effective electric fields and vice versa. At time $0.3 \mathrm{~ms}$, this effect has faded out, except in the vicinity of $l=6.1 \mathrm{~mm}$ where the membrane potential remains about $-45 \mathrm{mV}$, see the green solid line. This depolarization becomes even stronger $100 \mu$ s later, when values up to $20 \mathrm{mV}$ are achieved. Once such an action potential arises, it

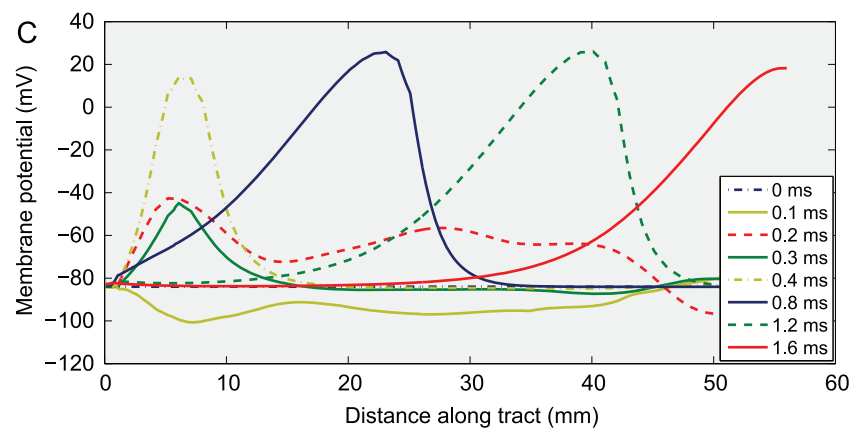

Figure 7. Membrane potential variation along the neural trajectory, for seven different time points. The same information as the white vertical lines of figure $5(\mathrm{C})$ is plotted as a function of space.

is conducted unattenuated down the axon. Figure $8(\mathrm{C})$ illustrates how the open probability of the activation gate $m$ increases to nearly $1(0.9993)$ shortly after the stimulation pulse, opening the sodium channels such that $\mathrm{Na}^{+}$ions flow into the cell and raise the membrane potential further providing positive feedback. Then, the open probability of the inactivation gate $h$ starts to decrease to nearly $0(0.0081)$, turning off the $\mathrm{Na}^{+}$flow. This decreased flow is also due to a reduced driving force $\left(V-V_{\mathrm{Na}}\right)$. Finally, the open probability of the activation gate $n$ of the potassium channel increases slowly and $\mathrm{K}^{+}$ions flow out of the cell. This causes the membrane potential to return gradually to its resting value. It takes longer than $2.5 \mathrm{~ms}$ for the gating variables to return to their resting values.

When increasing the stimulator output from 75.90 $\mathrm{A} \mu \mathrm{s}^{-1}$

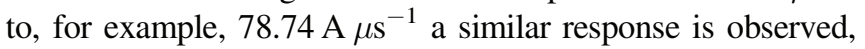
except that the action potential initiation occurs slightly faster, see case D of figure 5 . Once initiated, at the same position $l=6.1 \mathrm{~mm}$, it propagates towards the end of the axon at the

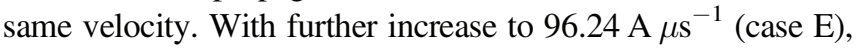
there arises a second action potential at $l=27.5 \mathrm{~mm}$ (again at a node of Ranvier). This signal is conducted in both directions of 

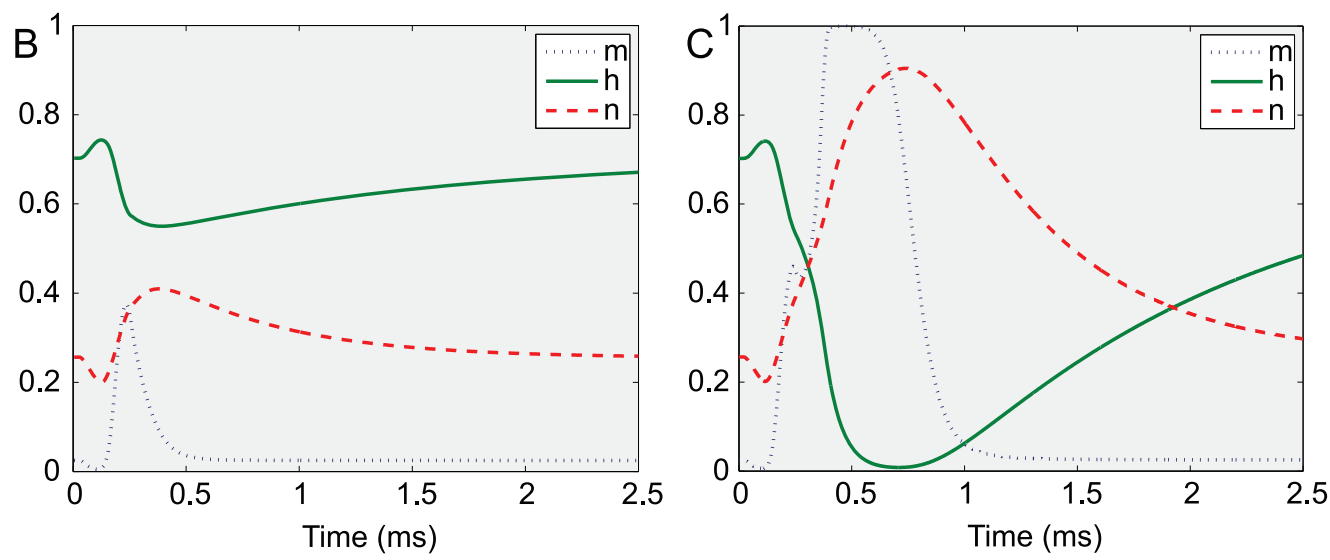

Figure 8. Variation of the dimensionless gating variables $m, h$ and $n$ at tract point $l=6.1 \mathrm{~mm}$ for cases $\mathrm{B}$ and $\mathrm{C}$ of figure 5 .

Table 3. Sensitivity analysis of the neural parameter values on the stimulation threshold and site. The stimulation threshold is defined as the minimal peak excitation current's time derivative needed to initiate an action potential at the selected tract. The changes are expressed in terms of percentage compared to the original threshold of $75.90 \mathrm{~A} \mathrm{\mu \textrm {s } ^ { - 1 }}$. The geometrical and electrical parameters were varied one at a time.

\begin{tabular}{|c|c|c|c|}
\hline Parameter & Range & Change (\%) & Site $(\mathrm{mm})$ \\
\hline$V_{0}{ }^{\mathrm{a}}$ & $-120 \rightarrow 40 \mathrm{mV}$ & $33.1 \rightarrow /$ & $7.1 \rightarrow /$ \\
\hline$R_{\mathrm{a}}$ & $0.1 \rightarrow 1 \Omega \mathrm{m}$ & $-38.6 \rightarrow 75.2$ & $8.1 \rightarrow 4.2$ \\
\hline Dendrite $L$ & $1 \rightarrow 2.2 \mathrm{~mm}$ & $0 \rightarrow 11.0$ & $6.1 \rightarrow 6.1$ \\
\hline Dendrite $d$ & $2 \rightarrow 32 \mu \mathrm{m}$ & $0.1 \rightarrow-1.4$ & $6.1 \rightarrow 6.1$ \\
\hline Initial segment $L$ & $1.5 \rightarrow 60 \mu \mathrm{m}$ & $-0.6 \rightarrow 1.4$ & $6.1 \rightarrow 6.1$ \\
\hline Unmyelin. axon $d^{\mathrm{b}}$ & $2.2 \rightarrow 10.2 \mu \mathrm{m}$ & $37.2 \rightarrow-15.3$ & $5.2 \rightarrow 7.1$ \\
\hline Myelin. internode $C_{\mathrm{m}}$ & $20 \rightarrow 50 \mu \mathrm{F} \mathrm{m}^{-2}$ & $-30.5 \rightarrow 0$ & $7.1 \rightarrow 6.1$ \\
\hline Myelin. internode $G_{\mathrm{m}}$ & $0.1 \rightarrow 0.2 \mathrm{~S} \mathrm{~m}^{-2}$ & $0 \rightarrow-16.7$ & $6.1 \rightarrow 6.1$ \\
\hline$C_{\mathrm{m}}$ of other segments & $9 \rightarrow 28 \mathrm{mF} \mathrm{m}^{-2}$ & $-18.2 \rightarrow 0$ & $6.1 \rightarrow 6.1$ \\
\hline \multicolumn{4}{|c|}{$\begin{array}{l}\text { a } \text { The initial } m_{0}, h_{0} \text { and } n_{0} \text { change with changing } V_{0}, \text { according to } \\
\alpha_{i}\left(V_{0}\right) /\left(\alpha_{i}\left(V_{0}\right)+\beta_{i}\left(V_{0}\right)\right) \text {. They equal } 7.565 \times 10^{-4}, 0.9954 \text { and } 8.846 \times 10^{-12} \\
\text { for }-120 \mathrm{mV} \text { and } 0.9999,2.4716 \times 10^{-6} \text { and } 0.999975 \text { for } 40 \mathrm{mV} \text {, respectively. } \\
\text { This range is equivalent to the combination of an initial diameter of the axon } \\
\text { hillock of } 4.4 \rightarrow 20.4 \mu \mathrm{m} \text {, a final diameter of the axon hillock of } 2.2 \rightarrow 10.2 \mu \mathrm{m} \text {, a } \\
\text { diameter of the initial segment of } 2.2 \rightarrow 10.2 \mu \mathrm{m} \text {, a diameter of the myelinated } \\
\text { internodes of } 5.0 \rightarrow 15.0 \mu \mathrm{m} \text { and a diameter of the nodes of Ranvier of } 2.2 \rightarrow \\
10.2 \mu \mathrm{m} \text {. }\end{array}$} \\
\hline
\end{tabular}

the fiber tract and collides with the other one at $l=20.5 \mathrm{~mm}$. Because action potentials are followed by a refractory period, the membrane is extensively hyperpolarized and remains as such until the ionic concentrations rebalance. As a consequence, if two propagating action potentials collide they annihilate. The main result of an increased stimulator output is faster communication. Due to the generation of multiple action potentials along the white matter fiber tract, the electric signal reaches faster the end of the axon, ready for synaptic interaction with other neurons of the brain network. This is also demonstrated by the last case $\mathrm{F}$ of figure 5 , in which the sti-

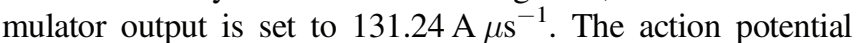
reaches the end of the axon at time $t=0.58 \mathrm{~ms}$.

\subsection{Sensitivity analysis of the neural parameter values}

The values of several geometrical and electrical parameters used in the model are not well known or may have a large inter- and/or intra-subject variability. In order to evaluate their influence on the predicted stimulation threshold, a sensitivity analysis is performed. This threshold is defined as the minimal peak excitation current's time derivative $\mathrm{d} I_{\text {coil }} / \mathrm{d} t$ needed to initiate an action potential at the selected tract. The parameters are varied according to the values found in literature (Rattay 1999, Wesselink et al 1999, Gentet et al 2000, McIntyre et al 2002, Manola et al 2007). The difference between the stimulation threshold, obtained with these extreme values, and the original threshold of $75.90 \mathrm{~A} \mu \mathrm{s}^{-1}$, corresponding with the parameter values of table A.1, are listed in terms of percentage in table 3. For example, Gentet et al (2000) estimated the membrane capacitance per unit area $C_{\mathrm{m}}$ for all segments, except the myelinated internodes, of cortical pyramidal neurons to be $0.009 \mathrm{~F} \mathrm{~m}^{-2}$. Applying this value decreases the stimulation threshold with $18.2 \%$ in comparison to the original used $C_{\mathrm{m}}$ of $0.028 \mathrm{~F} \mathrm{~m}^{-2}$. The results of this analysis demonstrate that the stimulation threshold is highly sensitive to the intracellular resistivity $R_{\mathrm{a}}$, with changes up to $75 \%$, whereas it is 
less sensitive to changes of lengths and diameters of the different segments. The initial state of the membrane potential $V_{0}$ also has a great effect on the stimulation threshold. Assuming a lower $V_{0}$ of $-120 \mathrm{mV}$ increases the threshold with $33.1 \%$ in comparison to the original $-84 \mathrm{mV}$. When assuming an initial membrane potential of $40 \mathrm{mV}$, our model was not able to generate an action potential, no matter how strong the stimulus is, due to the refractory period. The neuron cannot be activated again for some time since the inactivation variable $h$ has not recovered yet. Unlike other parameters, one extreme $V_{0}$ value does not correspond to a decrease, and the other with an increase of the obtained stimulation threshold. For example, an initial membrane potential of $-70 \mathrm{mV}$ results in an increase of $39.8 \%$ as well. The optimal value to start with is namely the resting potential $V_{\mathrm{r}}$, equal to $-84 \mathrm{mV}$.

The effect of the different model parameters on the stimulation site of the selected tract is also investigated. For the reference case, simulated with the parameter values of table A.1, an action potential originated at position $l=6.1 \mathrm{~mm}$, at a node of Ranvier. The last column of table 3 shows some parameter changes shifted this position. However, these shifts were limited $(\leqslant 2 \mathrm{~mm})$ and the positions always corresponded to nodes of Ranvier, one or two myelinated internodes closer or further from the M1 ROI. The uncertainty of the intracellular resistivity value $R_{\mathrm{a}}$ had the greatest influence on the stimulation site. Changing the length and diameter of the dendrite and the length of the initial segment did not alter the position. Though, it should be noted the stimulation threshold was also not that sensitive (less than 11\%) to these changes. Increasing the membrane conductance per unit area $G_{\mathrm{m}}$ of the myelinated internodes to $0.2 \mathrm{~S} \mathrm{~m}^{-2}$ did not affect the stimulation site, nor did the decrease of $C_{\mathrm{m}}$ of the other segments to $9 \mathrm{mF} \mathrm{m}^{-2}$. The latter two, however, resulted in a larger change of the stimulation threshold $(-16.7 \%$ and $-18.2 \%)$ than the increase of the diameter of the unmyelinated axon $(-15.3 \%)$. Thus, there is no strict linear relationship between the changed stimulation threshold and site.

At last, we remark the low sensitivity of the compartmental simulations to $\Delta t$. The time step can be taken up to $5 \mu \mathrm{s}$ instead of $1 \mu \mathrm{s}$, which would make the computational time five times shorter.

\subsection{Spatio-temporal variation of the membrane potentials}

Up to here we focused on a single tract, indicated in blue on figure 1. However, a group of 54 neural fiber tracts near the stimulated left M1 hand area were selected. Figure 9 illustrates their position relative to the T1-weighted image and the DTI color map of the subject's head. The coronal view is displayed in figure 10 , together with the simulated spatio-temporal variation of the membrane potentials for an applied stimulator output of $87.50 \mathrm{~A} \mu \mathrm{s}^{-1}$. Compare these images with figure 1, where the induced effective electric field is presented at the start of the stimulation pulse, that is $t=20 \mu \mathrm{s}$. Those white matter fiber tracts exposed to a large field will effectively generate more easily an action potential and conduct it unattenuated down their axons. These results suggest that for tracts
Table A.1. Geometrical and electrical parameter values of the different neural segments, based on human sensory fiber data (Wesselink et al 1999, Salvador 2009).

\begin{tabular}{|c|c|c|}
\hline Segment & Property & Value \\
\hline General & $\begin{array}{c}V_{\mathrm{r}} \\
V_{\mathrm{Na}} \\
V_{\mathrm{K}} \\
V_{\mathrm{L}} \\
G_{\mathrm{Na}} \\
G_{\mathrm{K}} \\
G_{\mathrm{L}} \\
R_{\mathrm{a}}\end{array}$ & $\begin{array}{c}-84 \times 10^{-3} \mathrm{~V} \\
43.7 \times 10^{-3} \mathrm{~V} \\
-84 \times 10^{-3} \mathrm{~V} \\
-84.14 \times 10^{-3} \mathrm{~V} \\
30000 \mathrm{~S} \mathrm{~m}^{-2} \\
300 \mathrm{~S} \mathrm{~m}^{-2} \\
600 \mathrm{~S} \mathrm{~m}^{-2} \\
0.33 \Omega \mathrm{m}\end{array}$ \\
\hline $\begin{array}{l}\text { Dendrite } \\
\text { (Passive) }\end{array}$ & $\begin{array}{c}L \\
d \\
C_{\mathrm{m}} \\
G_{\mathrm{m}}\end{array}$ & $\begin{array}{c}1000 \mu \mathrm{m} \\
8 \mu \mathrm{m} \\
0.028 \mathrm{~F} \mathrm{~m}^{-2} \\
2.73 \mathrm{~S} \mathrm{~m}^{-2}\end{array}$ \\
\hline $\begin{array}{l}\text { Soma } \\
\text { (Passive) }\end{array}$ & $\begin{array}{c}L \\
\text { Initial } d \\
\text { Final } d \\
C_{\mathrm{m}} \\
G_{\mathrm{m}}\end{array}$ & $\begin{array}{c}80 \mu \mathrm{m} \\
8 \mu \mathrm{m} \\
60 \mu \mathrm{m} \\
0.028 \mathrm{~F} \mathrm{~m}^{-2} \\
2.73 \mathrm{~S} \mathrm{~m}^{-2}\end{array}$ \\
\hline $\begin{array}{l}\text { Axon hillock } \\
\text { (Active) }\end{array}$ & $\begin{array}{c}L \\
\text { Initial } d \\
\text { Final } d \\
C_{\mathrm{m}}\end{array}$ & $\begin{array}{c}10 \mu \mathrm{m} \\
12 \mu \mathrm{m} \\
6 \mu \mathrm{m} \\
0.028 \mathrm{~F} \mathrm{~m}^{-2}\end{array}$ \\
\hline $\begin{array}{l}\text { Initial segment } \\
\text { (Active) }\end{array}$ & $\begin{array}{c}L \\
d \\
C_{\mathrm{m}}\end{array}$ & $\begin{array}{c}20 \mu \mathrm{m} \\
6 \mu \mathrm{m} \\
0.028 \mathrm{~F} \mathrm{~m}^{-2}\end{array}$ \\
\hline $\begin{array}{l}\text { Myelin. internode } \\
\text { (Passive) }\end{array}$ & $\begin{array}{c}L \\
d \\
C_{\mathrm{m}} \\
G_{\mathrm{m}}\end{array}$ & $\begin{array}{c} \pm 1000 \mu \mathrm{m}^{\mathrm{a}} \\
10 \mu \mathrm{m} \\
0.00005 \mathrm{~F} \mathrm{~m}^{-2} \\
0.1 \mathrm{~S} \mathrm{~m}^{-2}\end{array}$ \\
\hline $\begin{array}{l}\text { Ranvier node } \\
\text { (Active) }\end{array}$ & $\begin{array}{c}L \\
d \\
C_{\mathrm{m}}\end{array}$ & $\begin{array}{c}1.5 \mu \mathrm{m} \\
6 \mu \mathrm{m} \\
0.028 \mathrm{~F} \mathrm{~m}^{-2}\end{array}$ \\
\hline
\end{tabular}

a Depending on the length of the tract.

in order to be excited it is not sufficient for them to be close to the coil. It is an interplay of various factors, such as the position and orientation of the TMS coil, the applied excitation, the neural trajectory in relation to this coil and its course along the white and gray matter interface. For the complete progress in time of the membrane potentials, we refer to a movie that can be found online as supplementary data.

\subsection{Discussion}

Close to the stimulated ROI, here the left M1 hand area, a group of white matter fiber tracts is reconstructed using DTIbased tractography. Along these bundles the effective electric field was computed together with the spatiotemporal variation of the membrane potentials. We believe that this coupling of modeling of electromagnetism and neurophysiology is a suitable attempt of simulating the effect of TMS in space and over time as realistic as possible. It is important to note the different spatial and temporal scales. Whereas the electromagnetic computations are performed on a head model with $1-2 \mathrm{~mm}$ resolution, the neuronal responses are studied along the $(50-122 \mathrm{~mm}$ long) 


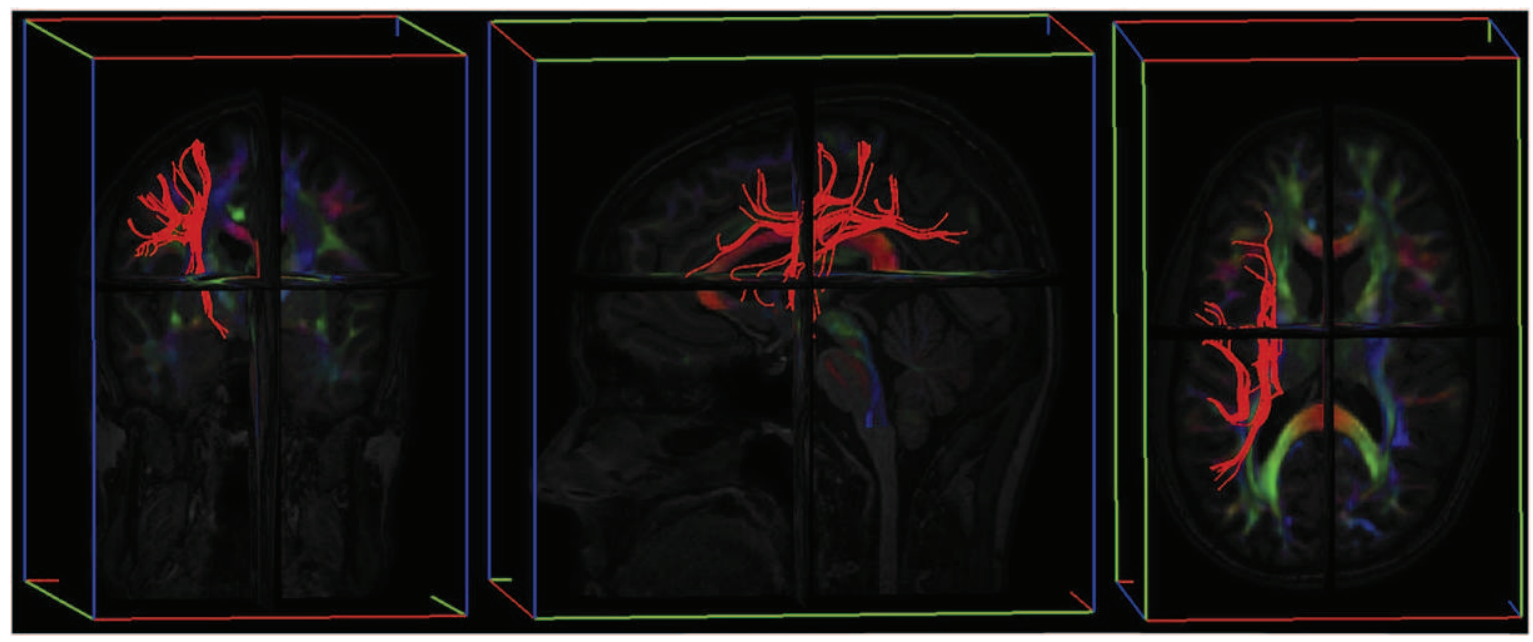

Figure 9. Position of the 54 white matter fiber tracts, in red, relative to the T1-weighted image fused with the DTI color map. The DTI colors indicate the dominant direction of the fibers: red (right to left, $x$-axis), green (posterior to anterior, $y$-axis) and blue (inferior to superior, $z$ axis). Coronal, sagittal and axial views, respectively.
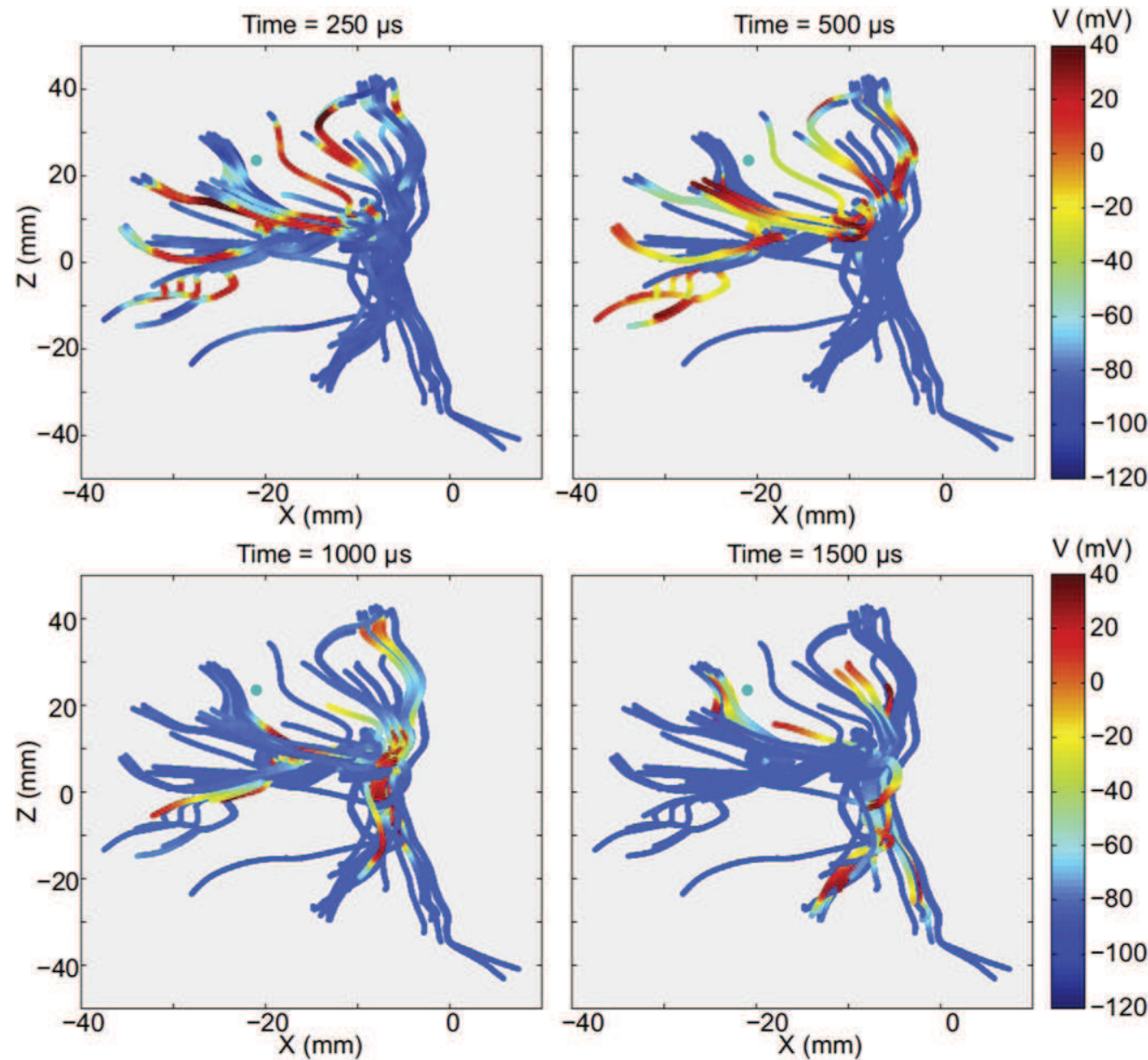

Figure 10. Temporal variation of the membrane potentials in all 54 tracts as response to a biphasic TMS pulse to the left M1 hand area. The blue dot is the seed ROI center. 
tracts with a variable $\mu \mathrm{m}$ mesh $(0.1-100 \mu \mathrm{m}$, see table 2$)$. Although the excitation current through the TMS coil was modeled with a pulse width of $230 \mu \mathrm{s}$, it gives rise to responses lasting longer than several ms, as seen in figure 10. This way, our proposed coupled model might offer a solution to explain the clinical observations of TMS effects propagating beyond the targeted site and lasting longer than the duration of the stimulation itself (Fox et al 2012, 2013, 2014, Sollmann et al 2015).

We demonstrated how an increasing stimulator output influences the membrane potentials and could deduce the threshold for activation. With further increase, we observed the generation of multiple action potentials along the fiber tract, causing the electric signal to reach the axonal end in less time. The sensitivity of the stimulation threshold and site towards the not well-known and inter- and/or intra-subject variable neural parameters appears to be acceptable for the case of the selected tract. However, future work should investigate more examples of white matter fiber tracts to extend and support this sensitivity analysis.

However, our model still has some important assumptions leaving room for improvement. The white matter fiber trajectories were reconstructed with DTI-based tractography using a deterministic streamline approach. With the current DTI resolution $(\geqslant 1 \mathrm{~mm})$, individual axons, neurons and synapses are not resolved, but the bulk-averaged tissue properties are derived for each voxel. During tractography, the dominant fiber orientation within each voxel is determined and successive discrete orientations provide an estimated tract reconstruction. This reconstruction is thus subject to noise contamination, which can lead to limited precision. The method might also fail to reconstruct the correct trajectories for crossing, kissing, branching or merging fibers (Tournier et al 2011). In these complex fiber configurations, the uncertainty of the dominant diffusion orientation is large, which makes it difficult to reconstruct white matter fiber pathways (Jeurissen et al 2013) with sufficient reliability. Further research is needed to tackle these limitations, for instance by resorting to probabilistic approaches (Behrens et al 2007, Jeurissen et al 2011). Moreover, the fiber tracts reconstructed with DTI-based tractography only represent anatomical or structural connectivity, thus no functional nor effective connectivity is included.

Furthermore, the compartmental neuron model has some limitations as well. The neural tracts are segmented in a single apical dendrite, soma, axon hillock, initial segment and a myelinated axon. These segments are represented by cylinders or truncated cones, as illustrated in figure 3. However, these are simplifications and in reality neurons have multiple dendrites which can be branching extensively. Some neurons have multiple axons and one axon features different diameters along its pathway. In the future, more realistic neuronal morphologies, as in (Kamitani et al 2001) and (Pashut et al 2011), should be incorporated. A distinction should also be made between the different types of neurons, such as the sensory neurons, the interneurons and pyramidal neurons. The electrical parameters of the neural segments, listed in table A.1, are obtained for pyramidal tract neurons (Wesselink et al 1999, Salvador et al 2011) but will differ for other neuron types, especially for cortical interneurons (Tsugorka et al 2007). Additionally, these parameter values are not well known and vary in the literature (Rattay 1999, Wesselink et al 1999, Gentet et al 2000, McIntyre et al 2002, Manola et al 2007). More accurate geometrical and morphological properties would lead to better estimates of the changes in membrane potentials due to stimulation. The initial state of all fiber tracts is assumed to be at rest, with membrane potential $V_{\mathrm{r}}$. A more accurate estimation of the actual brain state is needed, since table 3 demonstrates a high sensitivity of the stimulation threshold to the initial membrane potential. Resting state fMRI might offer a solution.

Moreover, no synapses are implemented for further communication to other neurons. Including this would allow not only to investigate the individual behavior of neural tracts, but also to predict the response on the whole brain network due to synaptic interactions between neurons. Our currently developed model can estimate how the electrical brain activity, considered to be initially in the resting state, is altered due to a TMS pulse by studying the spatio-temporal change of the membrane potentials of the neural tracts of interest. This is just the first step, which can also be noticed from the computed time span (a few ms). Once action potentials are triggered, they may propagate along the considered white matter fiber bundles. After being conducted down the axon they enter the axon terminals, which form the synapses with other neurons. When action potentials arrive at a pre-synaptic axon terminal, they cause a release of neurotransmitters into the synapse that bind to receptors in the post-synaptic neuron. New action potentials will be initiated, leading to further conduction of the neuronal signal, only if this post-synaptic neuron is excited sufficiently. This happens when multiple pre-synaptic neurons are triggered at the same time or when they release action potentials at a high frequency. In our model, a generated action potential is enabled to propagate in both directions of fiber tracts. The plasma membrane can indeed conduct the neuronal signals in both directions. However, synapse conducts the signal from the pre-synaptic axon terminal to the post-synaptic dendrite. Therefore, only those action potentials that are conducted down the axon into the terminal will contribute to further communication. In future work, implementing synapses and network interactions is essential to obtain a bigger picture and better understanding of the neuronal response to TMS.

We want to stress that our findings are obtained for an individual test subject and should not be extrapolated to other subjects or patients. The influence of the variability between brains and anatomical structures on the induced phenomena and corresponding simulation results needs to be taken into account.

\section{Conclusion}

In the past, TMS was thought to affect only the superficial cortical targets due to the limited penetration depth of the electric field of traditional stimulation coils. However, various 
recent clinical trials reported that TMS coils can modulate neuronal activity beyond the targeted site as well. We developed a computational model that indicates this deeper spread of electrical activity for the case of M1 hand area stimulation. Our presented model can approximate the impact of induced electric fields on neuronal structures, such as white matter fiber tracts used in this work. By mapping the electric fields along these fiber tracts, reconstructed through DTIbased tractography, we could gather model-based information on the behavior of membrane potentials in space and over time. This approach enables us to study the conceptual neurophysiological responses to TMS, impacting a distributed network of brain regions. Moreover, the use of neuronavigated TMS and MRI allows to treat each case and each subject or patient individually. This way, the gap between modeling and reality was decreased, increasing the fidelity of the developed model.

\section{Acknowledgments}

The authors gratefully acknowledge the financial support of the Agency for Innovation by Science and Technology in Flanders (IWT SB/101133), the Special Research Fund of Ghent University (BOF 13/24J/161) and the Fund of Scientific Research Flanders (FWO). The computations of this work were carried out using the Stevin Supercomputer Infrastructure at Ghent University.

\section{Appendix. Practical implementation of compartmental cable equation and applied parameter values}

In this work, we apply the Crank-Nicolson method to discretize the cable equation (1) in space and time with the grid steps $\Delta l$ and $\Delta t$, respectively

$$
\begin{aligned}
\frac{C_{\mathrm{m}}^{*}}{\Delta t}(V(l, t+\Delta t)-V(l, t)) \\
\quad+\frac{I_{\text {ion }}(l, t)+I_{\text {ion }}(l, t+\Delta t)}{2} \\
=G_{\mathrm{a}}^{*} \frac{V(l-\Delta l, t)+V(l-\Delta l, t+\Delta t)}{2} \\
\quad-2 G_{\mathrm{a}}^{*} \frac{V(l, t)+V(l, t+\Delta t)}{2} \\
\quad+G_{\mathrm{a}}^{*} \frac{V(l+\Delta l, t)+V(l+\Delta l, t+\Delta t)}{2} \\
\quad+G_{\mathrm{a}}^{*} \Delta l \frac{E_{l}(l-\Delta l, t)+E_{l}(l-\Delta l, t+\Delta t)}{2} \\
\quad-G_{\mathrm{a}}^{*} \Delta l \frac{E_{l}(l, t)+E_{l}(l, t+\Delta t)}{2} .
\end{aligned}
$$

Let us now include a staggered time grid, analogously to (Salvador 2009)

$$
f\left(l, t+\frac{\Delta t}{2}\right)=\frac{f(l, t)+f(l, t+\Delta t)}{2} .
$$

This brings equation (A.1) to the following expression:

$$
\begin{aligned}
& 2 \frac{C_{\mathrm{m}}^{*}}{\Delta t}\left(V\left(l, t+\frac{\Delta t}{2}\right)-V(l, t)\right)+I_{\text {ion }}\left(l, t+\frac{\Delta t}{2}\right) \\
& =G_{\mathrm{a}}^{*} V\left(l-\Delta l, t+\frac{\Delta t}{2}\right)-2 G_{\mathrm{a}}^{*} V\left(l, t+\frac{\Delta t}{2}\right) \\
& \quad+G_{\mathrm{a}}^{*} V\left(l+\Delta l, t+\frac{\Delta t}{2}\right) \\
& \quad+G_{\mathrm{a}}^{*} \Delta l E_{l}\left(l-\Delta l, t+\frac{\Delta t}{2}\right) \\
& \quad-G_{\mathrm{a}}^{*} \Delta l E_{l}\left(l, t+\frac{\Delta t}{2}\right)
\end{aligned}
$$

In matrix notation, we thus have:

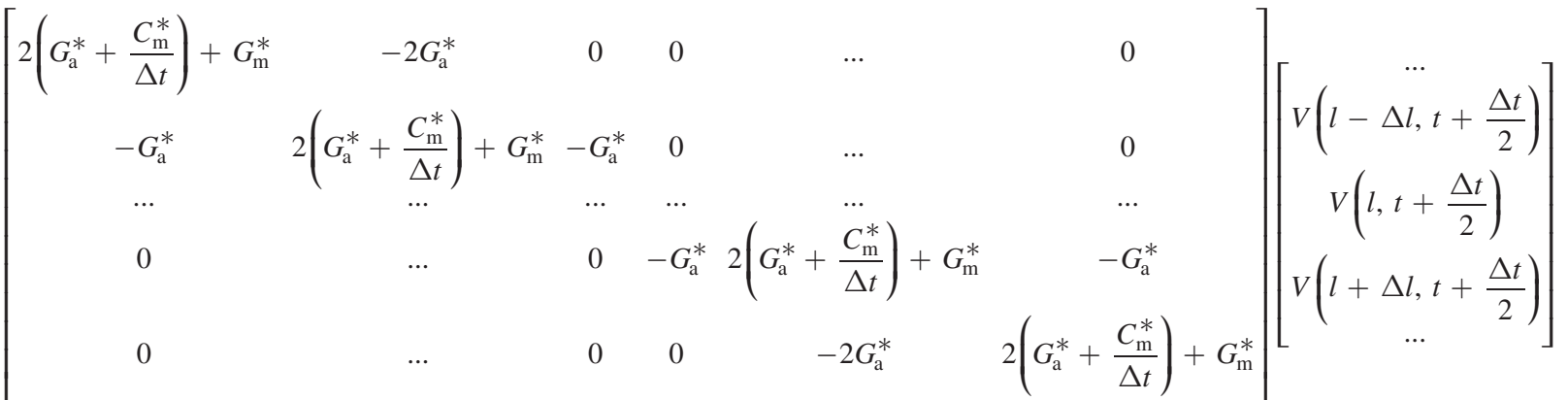

$$
\begin{aligned}
& =\left[\begin{array}{cccc}
2 \frac{C_{\mathrm{m}}^{*}}{\Delta t} & 0 & 0 & \ldots \\
0 & 2 \frac{C_{\mathrm{m}}^{*}}{\Delta t} & 0 & \ldots \\
0 & 0 & 2 \frac{C_{\mathrm{m}}^{*}}{\Delta t} & \ldots \\
\ldots & \ldots & \ldots & \ldots
\end{array}\right]\left[\begin{array}{c}
\ldots \\
V(l-\Delta l, t) \\
V(l, t) \\
V(l+\Delta l, t) \\
\ldots
\end{array}\right]+\left[\begin{array}{cccc}
G_{\mathrm{a}}^{*} \Delta l & -G_{\mathrm{a}}^{*} \Delta l & 0 & \ldots \\
G_{\mathrm{a}}^{*} \Delta l & -G_{\mathrm{a}}^{*} \Delta l & 0 & \ldots \\
0 & G_{\mathrm{a}}^{*} \Delta l & -G_{\mathrm{a}}^{*} \Delta l & \ldots \\
\ldots & \ldots & \ldots & \ldots
\end{array}\right]\left[\begin{array}{c}
\ldots \\
E_{l}\left(l-\Delta l, t+\frac{\Delta t}{2}\right) \\
E_{l}\left(l, t+\frac{\Delta t}{2}\right) \\
E_{l}\left(l+\Delta l, t+\frac{\Delta t}{2}\right) \\
\ldots
\end{array}\right]+\left[\begin{array}{c}
\ldots \\
V^{*}\left(l-\Delta l, t+\frac{\Delta t}{2}\right) \\
V^{*}\left(l, t+\frac{\Delta t}{2}\right) \\
V^{*}\left(l+\Delta l, t+\frac{\Delta t}{2}\right) \\
\ldots
\end{array}\right] .
\end{aligned}
$$


Depending on the segment at location $l$, whether it is assigned to the dendrites, soma, axon hillock, initial segment, Ranvier nodes or myelinated internodes, and thus whether it has passive or active properties, the parameter values vary as follows:

$$
\begin{gathered}
G_{\mathrm{m}}^{*}=\left\{\begin{array}{cc}
\pi d \Delta l G_{\mathrm{m}} & \text {; if passive } \\
\pi d \Delta l\left(G_{\mathrm{Na}} m^{3} h+G_{\mathrm{K}} n^{4}+G_{\mathrm{L}}\right) & ; \text { if active }
\end{array}\right. \\
V^{*}=\left\{\begin{array}{cc}
\pi d \Delta l G_{\mathrm{m}} V_{\mathrm{r}} & ; \text { if passive } \\
\pi d \Delta l\left(G_{\mathrm{Na}} m^{3} h V_{\mathrm{Na}}+G_{\mathrm{K}} n^{4} V_{\mathrm{K}}\right. & \\
\left.+G_{\mathrm{L}} V_{\mathrm{L}}\right) & ; \text { if active }
\end{array}\right.
\end{gathered}
$$

The membrane properties of the different segments, used in this work and similar to (Salvador 2009), are presented in table A.1. The used parameters for the expressions of $\alpha_{j}$ and $\beta_{j}$ are associated with the experimentally determined characteristics of human sensory fibers (Wesselink et al 1999). They were adjusted from the original $20{ }^{\circ} \mathrm{C}$ (Hodgkin and Huxley 1952) to a body temperature of $37^{\circ} \mathrm{C}$. The corresponding voltage-dependent transition rates are

$$
\begin{gathered}
\alpha_{m}=4.6 \times 10^{6} \frac{18.4 \times 10^{-3}+V}{1-\mathrm{e}^{\left(-18.4 \times 10^{-3}-V\right) / 10.3 \times 10^{-3}},}, \\
\beta_{m}=0.33 \times 10^{6} \frac{-22.7 \times 10^{-3}-V}{1-\mathrm{e}^{\left(22.7 \times 10^{-3}+V\right) / 9.16 \times 10^{-3}},} \\
\alpha_{h}=0.21 \times 10^{6} \frac{-111 \times 10^{-3}-V}{1-\mathrm{e}^{\left(111 \times 10^{-3}+V\right) / 11 \times 10^{-3}},} \\
\beta_{h}=14.1 \times 10^{3} \frac{1}{1+\mathrm{e}^{\left(-28.8 \times 10^{-3}-V\right) / 13.4 \times 10^{-3}},} \\
\alpha_{n}=51.7 \times 10^{3} \frac{93.2 \times 10^{-3}+V}{1-\mathrm{e}^{\left(-93.2 \times 10^{-3}-V\right) / 1.1 \times 10^{-3}}}, \\
\beta_{n}=92 \times 10^{3} \frac{-76 \times 10^{-3}-V}{1-\mathrm{e}^{\left(76 \times 10^{-3}+V\right) / 10.5 \times 10^{-3}}}
\end{gathered}
$$

and consequently, assuming the membrane is initially in rest with potential $V_{\mathrm{r}}$, the initial gating variables have the following values:

$$
\begin{aligned}
m_{0} & =0.02494, \\
h_{0} & =0.7026, \\
n_{0} & =0.2563 .
\end{aligned}
$$

With these values, $m, h$ and $n$ at the staggered grid can be calculated using the time discretized version of equation (6). For example, for the activation gate $m$ :

$$
\begin{aligned}
& m\left(l, t+\frac{\Delta t}{2}\right) \\
& =\frac{\alpha_{m}(l, t)+\left(\frac{1}{\Delta t}-\frac{\alpha_{m}(l, t)+\beta_{m}(l, t)}{2}\right) m\left(l, t-\frac{\Delta t}{2}\right)}{\frac{1}{\Delta t}+\frac{\alpha_{m}(l, t)+\beta_{m}(l, t)}{2}} .
\end{aligned}
$$

Subsequently, the parameters $G_{\mathrm{m}}^{*}$ and $V^{*}$ can be computed and the compartmental cable matrix equation (A.4) solved for the membrane potentials $V$ at time point $t+\frac{\Delta t}{2}$. Finally, these values can be converted back from the staggered to the normal grid $t+\Delta t$ using equation (A.2). This way of working produces a solution with an error of $O\left(\Delta t^{2}\right)$ while avoiding iteration of the equations (Salvador 2009).

We stress that the parameters listed in table A.1 and the pre-factors of equations (A.7)-(A.12) are not the only possible values, but rather averaged values over different neuron types that approximate reality as close as possible.

As can be seen from the matrix notation (A.4), the first and last equations satisfy the Von Neumann boundary conditions. They specify the first spatial derivative of the solution at the first and last points of the discretized neuron. These conditions occur naturally in neuronal modeling, since $\partial V / \partial l$ is proportional to the axial current through the neuron. It is often a well-considered assumption that no current is leaking out of the endpoints, thus stating Nagarajan et al (1993)

$$
\left.\frac{\partial V}{\partial l}\right|_{l=0}=\left.\frac{\partial V}{\partial l}\right|_{l=L_{\mathrm{tot}}}=0 ; \text { for all } t
$$

$L_{\text {tot }}$ is the total length of the fiber tract and so it determines the last point. They are usually called sealed-end boundary conditions and guarantee that charge can accumulate at each end. These boundary conditions are implemented by setting $\left.V\right|_{l=-1}=\left.V\right|_{l=1}$ and $\left.V\right|_{l=L_{\mathrm{tot}}+1}=\left.V\right|_{l=L_{\mathrm{tot}}-1}$; for all $t$,

as can be seen in the first and last equation of (A.4) (Niebur and Niebur 1991). The validity can be proven by expanding $V(l, t)$ in a Taylor series around the endpoints. For example, at $l=L_{\text {tot }}$,

$$
\begin{aligned}
\left.V\right|_{l=L_{\mathrm{tot}}+1}= & \left.V\right|_{l=L_{\mathrm{tot}}}+\left.\Delta l \frac{\partial V}{\partial l}\right|_{l=L_{\mathrm{tot}}} \\
& +\left.\frac{\Delta l^{2}}{2} \frac{\partial^{2} V}{\partial l^{2}}\right|_{l=L_{\mathrm{tot}}}+O\left(\Delta l^{3}\right) .
\end{aligned}
$$

Replacing $\quad \partial^{2} V /\left.\partial l^{2}\right|_{l=L_{\mathrm{tot}}}$ by $\quad\left(\left.V\right|_{l=L_{\mathrm{tot}}+1}-\left.2 V\right|_{l=L_{\mathrm{tot}}}+\right.$ $\left.\left.V\right|_{l=L_{\mathrm{tot}}-1}\right) / \Delta l^{2}$ and using equation (A.17), gives $\left.V\right|_{l=L_{\mathrm{tot}}+1}=\left.V\right|_{l=L_{\mathrm{tot}}-1}+O\left(\Delta l^{3}\right)$. Therefore, the applied implementation (A.18) is correct to the second order in $\Delta l$.

\section{References}

Basser P, Pajevic S, Pierpaoli C, Duda J and Aldroubi A 2000 In vivo fiber tractography using dt-mri data Magn. Reson. Med. 44 625-32

Behrens T, Berg H, Jbabdi S, Rushworth M and Woolrich M 2007 Probabilistic diffusion tractography with multiple fibre orientations: What can we gain? NeuroImage 34 144-55

Bestmann S, Baudewig J, Siebner H, Rothwell J and Frahm J 2004 Functional mri of the immediate impact of transcranial magnetic stimulation on cortical and subcortical motor circuits Eur. J. Neurosci. 19 1950-62 
Brighina F, Piazza A, Daniele O and Fierro B 2002 Modulation of visual cortical excitability in migraine with aura: effects of $1 \mathrm{~Hz}$ repetitive transcranial magnetic stimulation Exp. Brain Res. 145 177-81

Cahn S, Herzog A and Pascual-Leone A 2003 Paired-pulse transcranial magnetic stimulation: effects of hemispheric laterality, gender, and handedness in normal controls J. Clin. Neurophysiol. 20 371-4

Casarotto S, Lauro L, Bellina V, Casali A, Rosanova M, Pigorini A, Defendi S, Mariotti M and Massimini M 2010 Eeg responses to tms are sensitive to changes in the perturbation parameters and repeatable over time PLoS One 5 e10281

Cole K and Cole R 1941 Dispersion and absorption in dielectrics: alternating current characteristics J. Chem. Phys. 9 341-51

Crowther L, Marketos P, Williams P, Melikhov Y, Jiles D and Starzewski J 2011 Transcranial magnetic stimulation: improved coil design for deep brain investigation $J$. Appl. Phys. 109 07B314

Dannhauer M, Lanfer B, Wolters C and Knösche T 2011 Modeling of the human skull in eeg source analysis Hum. Brain Mapp. 32 1383-99

Davey K and Riehl M 2006 Suppressing the surface field during tms IEEE Trans. Biomed. Eng. 53 190-4

De Geeter N, Crevecoeur G and Dupré L 2011a Eddy-current simulations using an independent impedance method in anisotropic biological tissues IEEE Trans. Magn. 47 3845-8

De Geeter N, Crevecoeur G and Dupré L 2011b An efficient 3d eddy-current solver using an independent impedance method for transcranial magnetic stimulation IEEE Trans. Biomed. Eng. 58 310-20

De Geeter N, Crevecoeur G, Dupré L, Van Hecke W and Leemans A 2012 A dti-based model for tms using the independent impedance method with frequency-dependent tissue parameters Phys. Med. Biol. 57 2169-88

De Geeter N, Crevecoeur G, Leemans A and Dupré L 2014 Effective electric fields along realistic dti-based neural trajectories for modelling the stimulation mechanisms of tms Phys. Med. Biol. 60 453-71

De Lucia M, Parker G, Embleton K, Newton J and Walsh V 2007 Diffusion tensor mri-based estimation of the influence of brain tissue anisotropy on the effect of transcranial magnetic stimulation NeuroImage 36 1159-70

Deng Z, Lisanby S and Peterchev A 2013 Electric field depthfocality tradeoff in transcranial magnetic stimulation: simulation comparison of 50 coil designs Brain Stimul. 6 1-3

Ferreri F, Pasqualetti P, Määttä S, Ponzo D, Ferrarelli F, Tononi G, Mervaala E, Miniussi C and Rossini P 2011 Human brain connectivity during single and paired pulse transcranial magnetic stimulation NeuroImage 54 90-102

Fox M, Buckner R, Liu H, Chakravarty M, Lozano A and Pascual-Leone A 2014 Resting-state networks link invasive and noninvasive brain stimulation across diverse psychiatric and neurological diseases PNAS 111 E4367-75

Fox M, Halko M, Eldaief M and Pascual-Leone A 2012 Measuring and manipulating brain connectivity with resting state functional connectivity magnetic resonance imaging (fcmri) and transcranial magnetic stimulation (tms) NeuroImage 62 2232-43

Fox M, Liu H and Pascual-Leone A 2013 Identification of reproducible individualized targets for treatment of depression with tms based on intrinsic connectivity NeuroImage 66 151-60

Friston K, Ashburner J, Kiebel S, Nichols T and Penny W 2007 Statistical Parametric Mapping: The Analysis of Functional Brain Images (San Diego, CA, USA: Academic)

Gabriel S, Lau R and Gabriel C 1996 The dielectric properties of biological tissues: III. Parametric models for the dielectric spectrum of tissues Phys. Med. Biol. 41 2271-93
Gentet L, Stuart G and Clements J 2000 Direct measurement of specific membrane capacitance in neurons Biophys. J. 79 314-20

Goodwin B and Butson C 2015 Subject-specific multiscale modeling to investigate effects of transcranial magnetic stimulation Neuromodulation: Technol. Neural Interface 12296

Hannula H, Neuvonen T, Savolainen P, Hiltunen J, Ma Y, Antila H, Salonen O, Carlson S and Pertovaara A 2010 Increasing topdown suppression from prefrontal cortex facilitates tactile working memory NeuroImage 49 1091-8

Heller L and van Hulsteyn D 1992 Brain stimulation using electromagnetic sources: theoretical aspects Biophys. J. 63 129-38

Hodgkin A and Huxley A 1952 A quantitative description of membrane current and its application to conduction and excitation in nerve $J$. Physiol. 117 500-44

Hoogendam J, Ramakers G and Di Lazzaro V 2010 Physiology of repetitive transcranial magnetic stimulation of the human brain Brain Stimul. 3 98-118

Ilmoniemi R, Ruohonen J and Karhu J 1999 Transcranial magnetic stimulation-a new tool for functional imaging of the brain Crit. Rev. Biomed. Eng. 27 241-84

Ilmoniemi R, Virtanen J, Ruohonen J, Karhu J, Aronen H and Katila T 1997 Neuronal responses to magnetic stimulation reveal cortical reactivity and connectivity Neuroreport $83537-40$

Janssen A, Rampersad S, Lucka F, Lanfer B, Lew S, Aydin U, Wolters C, Stegeman D and Oostendorp T 2013 The influence of sulcus width on simulated electric fields induced by transcranial magnetic stimulation Phys. Med. Biol. 58 4881-96

Jeurissen B, Leemans A, Jones D, Tournier J and Sijbers J 2011 Probabilistic fiber tracking using the residual bootstrap with constrained spherical deconvolution Hum. Brain Mapp. 32 461-79

Jeurissen B, Leemans A, Tournier J, Jones D and Sijbers J 2013 Investigating the prevalence of complex fiber configurations in white matter tissue with diffusion magnetic resonance imaging Hum. Brain Mapp. 34 2747-66

Jones D and Leemans A 2011 Diffusion tensor imaging Methods Mol. Biol. 711 127-44

Julkunen P, Säisänen L, Danner N, Niskanen E, Hukkanen T, Mervaala E and Könönen M 2009 Comparison of navigated and non-navigated transcranial magnetic stimulation for motor cortex mapping, motor threshold and motor evoked potentials NeuroImage 44 790-5

Kähkönen S, Komssi S, Wilenius J and Ilmoniemi R 2005 Prefrontal transcranial magnetic stimulation produces intensity-dependent eeg responses in humans NeuroImage 24 955-60

Kamitani Y, Bhalodia V, Kubota Y and Shimojo S 2001 A model of magnetic stimulation of neocortical neurons Neurocomputing 38 697-703

Kim D, Georghiou G and Won C 2006 Improved field localization in transcranial magnetic stimulation of the brain with the utilization of a conductive shield plate in the stimulator IEEE Trans. Biomed. Eng. 53 720-5

Krieg S, Sabih J, Bulubasova L, Obermueller T, Negwer C, Janssen I, Shiban E, Meyer B and Ringel F 2014 Preoperative motor mapping by navigated transcranial magnetic brain stimulation improves outcome for motor eloquent lesions Neuro-Oncology 16 1274-82

Krieg T, Salinas F, Narayana S, Fox P and Mogul D 2015 Computational and experimental analysis of tms-induced electric field vectors critical to neuronal activation J. Neural Eng. 12046014

Leemans A, Jeurissen B, Sijbers J and Jones D 2009 Exploredti: a graphical toolbox for processing, analyzing, and visualizing diffusion mr data Proc. Intl. Soc. Mag. Reson. Med. (Hawaii, USA) 
Lioumis P, Kičić D, Savolainen P, Mäkelä J and Kähkönen S 2009 Reproducibility of tms-evoked eeg responses Hum. Brain Mapp. 30 1387-96

Loo C and Mitchell P 2005 A review of the efficacy of transcranial magnetic stimulation (tms) treatment for depression, and current and future strategies to optimize efficacy $J$. Affect. Disorders 3 255-67

Lu M, Ueno S, Thorlin T and Persson M 2008 Calculating the activating function in the human brain by transcranial magnetic stimulation IEEE Trans. Magn. 44 1438-41

Maeda F, Keenan J, Tormos J, Topka H and Pascual-Leone A 2000 Interindividual variability of the modulatory effects of repetitive transcranial magnetic stimulation on cortical excitability Exp. Brain Res. 133 425-30

Manola L, Holsheimer J, Veltink P and Buitenweg J 2007 Anodal versus cathodal stimulation of motor cortex: a modeling study Clin. Neurophysiol. 118 464-74

McIntyre C, Richardson A and Grill W 2002 Modeling the excitability of mammalian nerve fibers: influence of afterpotentials on the recovery cycle J. Neurophysiol. 87 995-1006 PMID: 11826063

Miranda P, Correia L, Salvador R and Basser P 2007 Tissue heterogeneity as a mechanism for localized neural stimulation by applied electric fields Phys. Med. Biol. 52 5603-17

Miranda P, Hallett M and Basser P 2003 The electric field induced in the brain by magnetic stimulation: a $3 \mathrm{~d}$ finite-element analysis of the effect of tissue heterogeneity and anisotropy IEEE Trans. Biomed. Eng. 50 1074-85

Nagarajan S, Durand M and Warman E 1993 Effects of induced electric fields on finite neuronal structures: a simulation study IEEE Trans. Biomed. Eng. 40 1175-88

Niebur E and Niebur D 1991 Numerical implementation of sealedend boundary conditions in cable theory IEEE Trans. Biomed. Eng. 38 1266-71

Opitz A, Windhoff M, Heidemann R, Turner R and Thielscher A 2011 How the brain tissue shapes the electric field induced by transcranial magnetic stimulation Neurolmage 58 849-59

Pascual-Leone A, Walsh V and Rothwell J 2000 Transcranial magnetic stimulation in cognitive neuroscience-virtual lesion, chronometry, and functional connectivity Curr. Opin. Neurobiol. 10 232-7

Pashut T, Wolfus S, Friedman A, Lavidor M, Bar-Gad I, Yeshurun Y and Korngreen A 2011 Mechanisms of magnetic stimulation of central nervous system neurons PLoS Comput. Biol. 7 e1002022

Pasley B, Allen E and Freeman R 2009 State-dependent variability of neuronal responses to transcranial magnetic stimulation of the visual cortex Neuron 62 291-303

Picht T et al 2013 A comparison of language mapping by preoperative navigated transcranial magnetic stimulation and direct cortical stimulation during awake surgery Neurosurgery 72 808-19
Rattay F 1986 Analysis of models for external stimulation of axons IEEE Trans. Biomed. Eng. 10 974-7

Rattay F 1999 The basic mechanism for the electrical stimulation of the nervous system Neuroscience 89 335-46

Sack A and Linden D 2003 Combining transcranial magnetic stimulation and functional imaging in cognitive brain research: possibilities and limitations Brain Res. Rev. 43 41-56

Salinas F, Lancaster J and Fox P 2007 Detailed 3d models of the induced electric field of transcranial magnetic stimulation coils Phys. Med. Biol. 52 2879-92

Salvador R 2009 Numerical modelling in transcranial magnetic stimulation PhD Thesis University of Lisbon, Portugal

Salvador R, Silva S, Basser P and Miranda P 2011 Determining which mechanisms lead to activation in the motor cortex: a modeling study of transcranial magnetic stimulation using realistic stimulus waveforms and sulcal geometry Clin. Neurophysiol. 122 748-58

Shahid S, Bikson M, Salman H, Wen P and Ahfock T 2014 The value and cost of complexity in predictive modelling: role of tissue anisotropic conductivity and fibre tracts in neuromodulation J. Neural Eng. 11036002

Silva S, Basser P and Miranda P 2008 Elucidating the mechanisms and loci of neuronal excitation by transcranial magnetic stimulation using a finite element model of a cortical sulcus Clin. Neurophysiol. 119 2405-13

Silvanto J and Muggleton N 2008 New light through old windows: moving beyond the virtual lesion approach to transcranial magnetic stimulation NeuroImage 39 549-52

Sollmann N, Giglhuber K, Tussis L, Meyer B, Ringel F and Krieg S 2015 ntms-based dti fiber tracking for language pathways correlates with language function and aphasia-a case report Clin. Neurol. Neurosur. $13625-8$

Thielscher A and Kammer T 2004 Electric field properties of two commercial figure- 8 coils in tms: calculation of focality and efficiency Clin. Neurophysiol. 115 1697-708

Thut G and Pascual-Leone A 2010 A review of combined tms-eeg studies to characterize lasting effects of repetitive tms and assess their usefulness in cognitive and clinical neuroscience Brain Topogr. 22 219-32

Tournier J, Mori S and Leemans A 2011 Diffusion tensor imaging and beyond Magn. Reson. Med. 65 1532-56

Tsugorka T, Dovgan O, Stepanyuk A and Cherkas V 2007 Variety of types of cortical interneurons Neurophysiology $39227-36$

Wesselink W, Holsheimer J and Boom H 1999 A model of the electrical behaviour of myelinated sensory nerve fibres based on human data Med. Biol. Eng. Comput. 37 228-35

Zangen A, Roth Y, Voller B and Hallett M 2005 Transcranial magnetic stimulation of deep brain regions: evidence for efficacy of the h-coil Clin. Neurophysiol. $116775-9$ 